\title{
Relaxed constant positive linear dependence constraint qualification and its application to bilevel programs
}

\author{
Mengwei $\mathrm{Xu}^{*}$ and Jane J. Ye
}

\begin{abstract}
Relaxed constant positive linear dependence constraint qualification (RCPLD) for a system of smooth equalities and inequalities is a constraint qualification that is weaker than the usual constraint qualifications such as Mangasarian Fromovitz constraint qualification and the linear constraint qualification. Moreover RCPLD is known to induce an error bound property. In this paper we extend RCPLD to a very general feasibility system which may include Lipschitz continuous inequality constraints, complementarity constraints and abstract constraints. We show that this RCPLD for the general system is a constraint qualification for the optimality condition in terms of limiting subdifferential and limiting normal cone and it is a sufficient condition for the error bound property under the strict complementarity condition for the complementarity system and Clarke regularity conditions for the inequality constraints and the abstract constraint set. Moreover we introduce and study some sufficient conditions for RCPLD including the relaxed constant rank constraint qualification (RCRCQ). Finally we apply our results to the bilevel program.
\end{abstract}

Key Words. Constraint qualification, error bound property, nonsmooth program, RCPLD, variational analysis, complementarity system, bilevel program.

2010 Mathematics Subject Classification. 49J52, 90C31, 90C33.

\footnotetext{
*School of Mathematics, Tianjin University, Tianjin, 300072, China. E-mail: xumengw@hotmail.com. The research of this author was supported by the National Natural Science Foundation of China under Projects No. 11601376

${ }^{\dagger}$ Corresponding author. Department of Mathematics and Statistics, University of Victoria, Victoria, B.C., Canada V8W 2Y2. E-mail: janeye@uvic.ca. The research of this author was partially supported by NSERC.
} 


\section{Introduction}

A constraint qualification is a condition imposed on the constraint region of a mathematical program under which the Karush-Kuhn-Tucker (KKT) condition holds at any local optimal solution. Other than guaranteeing KKT condition holding at all local optimal solutions, some constraint qualifications also lead to existence of error bounds to the feasible region and hence play a key role in convergence analysis of certain computational methods. Hence studying constraint qualifications is essential in both theoretical and numerical points of view.

For smooth nonlinear programs with equality and inequality constraints, the classical constraint qualifications are the linear independence constraint qualification (LICQ), MangasarianFromovitz constraint qualification (MFCQ), and the linear constraint qualification (LCQ), i.e., all functions in the equality and inequality constraints are affine. These three classical constraint qualifications may be too restrictive for many problems. Janin [16] relaxed LICQ and proposed the constant rank constraint qualification (CRCQ), which neither implies nor is implied by MFCQ. The concept of CRCQ was weakened to the relaxed constant rank constraint qualification (RCRCQ) which is shown to be a constraint qualification by Minchenko and Stakhovshi [19]. Qi and Wei [26] introduced the concept of the constant positive linear dependence (CPLD) condition which is weaker than both CRCQ and MFCQ. CPLD was shown to be a constraint qualification by Andreani, Martínez and Schuverdt in [2].

In [1, Andreani, Haeser, Schuverdt and Silva introduced the following relaxed version of CPLD for a system of smooth equality and inequality constraints:

$$
g_{i}(x) \leq 0, i=1, \cdots, n, \quad h_{i}(x)=0, i=1, \cdots, m,
$$

where $g_{i}, h_{i}: \mathbb{R}^{d} \rightarrow \mathbb{R}$ are smooth at $x^{*}$, a feasible solution. $x^{*}$ is said to satisfy the relaxed constant positive linear dependence constraint qualification (RCPLD) if there exists $\mathbb{U}\left(x^{*}\right)$, a neighborhood of $x^{*}$ such that

(i) $\left\{\nabla h_{i}(x)\right\}_{i=1}^{m}$ has the same rank for every $x \in \mathbb{U}\left(x^{*}\right)$.

(ii) Let $J \subseteq\{1, \cdots, m\}$ be such that $\left\{\nabla h_{i}\left(x^{*}\right)\right\}_{i \in J}$ is a basis for $\operatorname{span}\left\{\nabla h_{i}\left(x^{*}\right)\right\}_{i=1}^{m}$. For every $I \subseteq I_{g}^{*}:=\left\{i: g_{i}\left(x^{*}\right)=0\right\}$, if $\left\{\nabla g_{i}\left(x^{*}\right)\right\}_{i \in I} \cup\left\{\nabla h_{i}\left(x^{*}\right)\right\}_{i \in J}$ is positive linearly dependent, i.e., there exist scalars $\lambda_{i} \geq 0, i \in I, \mu_{i}, i \in J$ not all zero such that

$$
0=\sum_{i \in I} \lambda_{i} \nabla g_{i}\left(x^{*}\right)+\sum_{i \in J} \mu_{i} \nabla h_{i}\left(x^{*}\right),
$$

then $\left\{\nabla g_{i}(x)\right\}_{i \in I} \cup\left\{\nabla h_{i}(x)\right\}_{i \in J}$ is linearly dependent for every $x \in \mathbb{U}\left(x^{*}\right)$.

It is easy to see that in the case where either LCQ or MFCQ holds, RCPLD also holds. Hence RCPLD is weaker than LCQ and MFCQ. In [1], the authors not only showed that RCPLD is a 
constraint qualification but also proved that if all functions $g_{i}, h_{i}$ have second-order derivatives at all points near the point $x^{*}$, then RCPLD is a sufficient condition for the error bound property: $\exists \alpha>0, \mathbb{U}\left(x^{*}\right)$, a neighborhood of $x^{*}$ such that

$$
d_{\mathcal{F}}(x) \leq \alpha\left(\left\|g_{+}(x)\right\|+\|h(x)\|\right), \quad \forall x \in \mathbb{U}\left(x^{*}\right)
$$

where $\mathcal{F}:=\{x \mid g(x) \leq 0, h(x)=0\}, g(x)=\left(g_{1}(x), \ldots, g_{n}(x)\right), h(x)=\left(h_{1}(x), \ldots, h_{m}(x)\right), d_{\mathcal{F}}(x)$ is the distance from $x$ to set $\mathcal{F},\|\cdot\|$ denotes any norm and $g_{+}(x):=\max \{0, g(x)\}$, where the maximum is taken component-wise. Moreover as an open question, in [1], a question was asked on whether or not it was possible to prove the error bound property without imposing the second-order differentiability of all functions. In Guo, Zhang and Lin [14, it was shown that the error bound property holds under RCPLD without imposing the second order differentiability of all functions. Other than using it as a constraint qualification to ensure the KKT condition holds, RCPLD is also used in the convergence analysis of the augmented Lagrangian method to obtain a KKT point (see e.g., [1, 3, 15, 28]). Recently, Guo and Ye [13, Corollary 3] extended RCPLD to the case where there is an extra abstract constraint set and showed that it is still a constraint qualification. In [11, Definition 4.3], a version of RCPLD called MPEC RCPLD was introduced for the mathematical programs with equilibrium constraints (MPEC) and was shown in [10, Corollary 4.1] that it is a constraint qualification for M-stationary conditions. Moreover, it was shown in [14, Theorem 5.1] that the RCPLD for MPECs ensures the error bound property under the assumption of strict complementarity.

In this paper, we extend RCPLD to the following very general feasibility system:

$$
\begin{aligned}
& g_{i}(x) \leq 0, i=1, \cdots, n, \\
& h_{i}(x)=0, i=1, \cdots, m, \\
& (G(x), H(x)) \in \Omega^{p}, \\
& x \in C,
\end{aligned}
$$

where $\Omega^{p}:=\left\{(y, z) \in \mathbb{R}^{p} \times \mathbb{R}^{p} \mid 0 \leq y \perp z \geq 0\right\}$ is the $p$ th dimensional complementarity set, $C:=C_{1} \times C_{2} \times \cdots \times C_{l}$ with $C_{i} \subseteq \mathbb{R}^{q_{i}}$ closed, $i=1, \cdots, l, q_{1}+\cdots+q_{l}=d$, the functions $g_{i}: \mathbb{R}^{d} \rightarrow \mathbb{R}, i=1, \cdots, n$, are locally Lipschitz continuous and $h_{i}: \mathbb{R}^{d} \rightarrow \mathbb{R}, i=1, \cdots, m$, $G, H: \mathbb{R}^{d} \rightarrow \mathbb{R}^{p}$, are continuously differentiable at $x^{*}$, a feasible solution.

Denote the feasible region of system (1.1) by $\mathcal{F}$. For a feasible point $x^{*} \in \mathcal{F}$, we define the following index sets:

$$
\begin{aligned}
I_{g}^{*} & :=\left\{i=1, \cdots, n: g_{i}\left(x^{*}\right)=0\right\}, \\
\mathcal{I}^{*} & :=\left\{i=1, \cdots, p: 0=G_{i}\left(x^{*}\right)<H_{i}\left(x^{*}\right)\right\}, \\
\mathcal{J}^{*} & :=\left\{i=1, \cdots, p: 0=G_{i}\left(x^{*}\right)=H_{i}\left(x^{*}\right)\right\}, \\
\mathcal{K}^{*} & :=\left\{i=1, \cdots, p: G_{i}\left(x^{*}\right)>H_{i}\left(x^{*}\right)=0\right\} .
\end{aligned}
$$


Definition 1.1 (RCPLD for the nonsmooth system (1.1)) We say that the relaxed constant positive linear dependence constraint qualification (RCPLD) holds at $x^{*} \in \mathcal{F}$ for system (1.1) if the following conditions hold:

(i) The vectors $\left\{\nabla h_{i}(x)\right\}_{i=1}^{m} \cup\left\{\nabla G_{i}(x)\right\}_{i \in \mathcal{I}^{*}} \cup\left\{\nabla H_{i}(x)\right\}_{i \in \mathcal{K}^{*}}$ have the same rank for all $x$ in a neighbourhood of $x^{*}$.

(ii) Let $\mathcal{I}_{1} \subseteq\{1, \cdots, m\}, \mathcal{I}_{2} \subseteq \mathcal{I}^{*}, \mathcal{I}_{3} \subseteq \mathcal{K}^{*}$ be such that the set of vectors $\left\{\nabla h_{i}\left(x^{*}\right)\right\}_{i \in \mathcal{I}_{1}} \cup$ $\left\{\nabla G_{i}\left(x^{*}\right)\right\}_{i \in \mathcal{I}_{2}} \cup\left\{\nabla H_{i}\left(x^{*}\right)\right\}_{i \in \mathcal{I}_{3}}$ is a basis for

$$
\operatorname{span}\left\{\left\{\nabla h_{i}\left(x^{*}\right)\right\}_{i=1}^{m} \cup\left\{\nabla G_{i}\left(x^{*}\right)\right\}_{i \in \mathcal{I}^{*}} \cup\left\{\nabla H_{i}\left(x^{*}\right)\right\}_{i \in \mathcal{K}^{*}}\right\} .
$$

For any index sets $\mathcal{I}_{4} \subseteq I_{g}^{*}, \mathcal{I}_{5}, \mathcal{I}_{6} \subseteq \mathcal{J}^{*}$, if there exists a nonzero vector $\left(\lambda^{g}, \lambda^{h}, \lambda^{G}, \lambda^{H}, \eta^{*}\right) \in$ $\mathbb{R}^{n} \times \mathbb{R}^{m} \times \mathbb{R}^{p} \times \mathbb{R}^{p} \times \mathbb{R}^{d}$ satisfying $\lambda_{i}^{g} \geq 0$ for $i \in \mathcal{I}_{4}$, and either $\lambda_{i}^{G}>0, \lambda_{i}^{H}>0$ or $\lambda_{i}^{G} \lambda_{i}^{H}=$ $0, \forall i \in \mathcal{J}^{*}, \eta^{*}=\left(\eta_{1}^{*}, \cdots, \eta_{l}^{*}\right) \in \mathcal{N}_{C}\left(x^{*}\right)=\mathcal{N}_{C_{1}}\left(x_{1}^{*}\right) \times \cdots \times \mathcal{N}_{C_{l}}\left(x_{l}^{*}\right), v_{i}^{*} \in \partial g_{i}\left(x^{*}\right)$ for $i \in \mathcal{I}_{4}$ such that

$$
0=\sum_{i \in \mathcal{I}_{4}} \lambda_{i}^{g} v_{i}^{*}+\sum_{i \in \mathcal{I}_{1}} \lambda_{i}^{h} \nabla h_{i}\left(x^{*}\right)-\sum_{i \in \mathcal{I}_{2} \cup \mathcal{I}_{5}} \lambda_{i}^{G} \nabla G_{i}\left(x^{*}\right)-\sum_{i \in \mathcal{I}_{3} \cup \mathcal{I}_{6}} \lambda_{i}^{H} \nabla H_{i}\left(x^{*}\right)+\eta^{*},
$$

then for all $k$ sufficiently large, the set of vectors

$$
\left\{v_{i}^{k}\right\}_{i \in \mathcal{I}_{4}} \cup\left\{\nabla h_{i}\left(x^{k}\right)\right\}_{i \in \mathcal{I}_{1}} \cup\left\{\nabla G_{i}\left(x^{k}\right)\right\}_{i \in \mathcal{I}_{2} \cup \mathcal{I}_{5}} \cup\left\{\nabla H_{i}\left(x^{k}\right)\right\}_{i \in \mathcal{I}_{3} \cup \mathcal{I}_{6}} \cup\left\{\nu_{i}^{k}\right\}_{i \in L},
$$

where $v_{i}^{k} \in \partial g_{i}\left(x^{k}\right), \nu_{i}^{k}:=\{0\}^{s_{i}} \times\left\{\eta_{i}^{k}\right\} \times\{0\}^{t_{i}}$ with $\eta_{i}^{k} \in \mathcal{N}_{C_{i}}\left(x_{i}^{k}\right), s_{i}:=q_{1}+\cdots+q_{i-1}$, $t_{i}:=q_{i+1}+\cdots+q_{l}, L:=\left\{1, \ldots, l: \eta_{i}^{k} \neq 0\right\}$ and $x^{k} \neq x^{*}$, is linearly dependent for all sequences $\left\{x^{k}\right\},\left\{v^{k}\right\},\left\{\eta^{k}\right\}$ satisfying $x^{k} \rightarrow x^{*}, v_{i}^{k} \rightarrow v_{i}^{*}, \eta^{k}:=\left(\eta_{1}^{k}, \ldots, \eta_{l}^{k}\right)=\sum_{i \in L} \nu_{i}^{k} \rightarrow$ $\eta^{*}$ as $k \rightarrow \infty$.

Remark 1.1 In Definition 1.1 (ii), we use sequences instead of neighborhoods. Although we could also use a neighborhood in the definition equivalently, it is more convenient to use the sequential form since if the point $x^{*}$ is an isolated non-differentiable point, i.e., there exists a neighborhood around $x^{*}$ where $g$ is differentiable, then $v_{i}^{k}$ can be taken as the gradient $\nabla g_{i}\left(x^{k}\right)$. Since a Lipschitz continuous function is differentiable almost everywhere and so such points are abundant.

In the case where the rank of $\left\{\nabla h_{i}\left(x^{*}\right)\right\}_{i=1}^{m} \cup\left\{\nabla G_{i}\left(x^{*}\right)\right\}_{i \in \mathcal{I}^{*}} \cup\left\{\nabla H_{i}\left(x^{*}\right)\right\}_{i \in \mathcal{K}^{*}}$ is equal to $d$, it is easy to see that RCPLD holds automatically. What is more, in Theorem 4.1 we will show that the error bound property holds in this case.

Note that Definition 1.1 is weaker than the one defined in Guo and Ye [13, Corollary 3] for the system containing only smooth equality and inequality constraints and one abstract constraint, in which the stronger condition $\left\{\nabla g_{i}(x)\right\}_{i \in \mathcal{I}_{4}} \cup\left\{\nabla h_{i}(x)\right\}_{i \in \mathcal{I}_{1}}$ is linearly dependent for every $x \in \mathbb{U}\left(x^{*}\right)$ required. 
If the set of vectors in (1.2) is replaced by the following set of vectors

$$
\left\{v_{i}^{k}\right\}_{i \in \mathcal{I}_{4}} \cup\left\{\nabla h_{i}\left(x^{k}\right)\right\}_{i \in \mathcal{I}_{1}} \cup\left\{\nabla G_{i}\left(x^{k}\right)\right\}_{i \in \mathcal{I}_{2} \cup \mathcal{I}_{5}} \cup\left\{\nabla H_{i}\left(x^{k}\right)\right\}_{i \in \mathcal{I}_{3} \cup \mathcal{I}_{6}} \cup\left\{\eta^{k}\right\},
$$

then since $\eta^{k}=\sum_{i \in L} \nu_{i}^{k}$, the resulting condition would be stronger than the RCPLD defined in Definition 1.1. We illustrate this by Example 4.1.

In this paper we show that RCPLD for the nonsmooth feasibility system is a constraint qualification for any optimization problem with a Lipschitz objective function and the constraints described as in the feasibility system (1.1). Moreover with some extra conditions, we will show that RCPLD is a sufficient condition for the following error bound property: $\exists \alpha>0, \mathbb{U}\left(x^{*}\right)$, a neighborhood of $x^{*}$ such that

$$
d_{\mathcal{F}}(x) \leq \alpha\left(\left\|g_{+}(x)\right\|+\|h(x)\|+\sum_{i=1}^{p} d_{\Omega}\left(G_{i}(x), H_{i}(x)\right)\right), \quad \forall x \in \mathbb{U}\left(x^{*}\right) \cap C,
$$

where $\Omega:=\Omega^{1}=\left\{(y, z) \in \mathbb{R}^{2} \mid 0 \leq y \perp z \geq 0\right\}$.

One of the motivations to extend the concept of RCPLD to the nonsmooth system (1.1) is to study the constraint qualification and optimality condition for the following bilevel program:

$$
\begin{aligned}
(\mathrm{BP}) \quad \min & F(x, y) \\
\text { s.t. } & y \in S(x), G(x, y) \leq 0, H(x, y)=0,
\end{aligned}
$$

where $S(x)$ denotes the solution set of the lower level program

$$
\left(\mathrm{P}_{x}\right) \quad \min _{y \in Y(x)} f(x, y)
$$

and $Y(x):=\left\{y \in \mathbb{R}^{s}: g(x, y) \leq 0, h(x, y)=0\right\}, F: \mathbb{R}^{d} \times \mathbb{R}^{s} \rightarrow \mathbb{R}$ is locally Lipschitz continuous, $G: \mathbb{R}^{d} \times \mathbb{R}^{s} \rightarrow \mathbb{R}^{p}$ and $H: \mathbb{R}^{d} \times \mathbb{R}^{s} \rightarrow \mathbb{R}^{q}$ are continuously differentiable, $f: \mathbb{R}^{d} \times \mathbb{R}^{s} \rightarrow \mathbb{R}$, $g: \mathbb{R}^{d} \times \mathbb{R}^{s} \rightarrow \mathbb{R}^{m}, h: \mathbb{R}^{d} \times \mathbb{R}^{s} \rightarrow \mathbb{R}^{n}$ are continuously differentiable and twice continuously differentiable with respect to variable $y$.

Bilevel programs naturally fall in the domain of global optimization since in the lower level problem, the global optimal solution is always required in either optimality conditions or numerical algorithms. A popular approach to deal with (BP) is to replace the set of global solutions $S(x)$ by the KKT optimality conditions of the lower level problem. This reformulation is based on the fact that if the lower level problem $\left(P_{x}\right)$ is a convex program for each fixed $x$ and certain constraint qualification holds, then $y \in S(x)$ if and only if its KKT optimality condition holds. But due to the introduction of multipliers for the lower level problem as extra variables, the resulting reformulation may not be equivalent to the original bilevel program even in the case where $\left(P_{x}\right)$ is convex but the multipliers are not unique. For the discussion of this issue and the recent new results, the reader is referred to recent paper [31] and the reference within for more 
discussions. If the lower level problem $\left(P_{x}\right)$ is not a convex program for each fixed $x$, the KKT condition for the lower level problem is usually only a necessary but not sufficient condition for optimality and moreover, as pointed out by Mirrlees in [20], such a reformulation by the KKT condition may miss out the true optimal solution of the original bilevel program.

Instead of using $y \in S(x)$ as a constraint in (BP), Outrata 25] proposed to replace it with $f(x, y)-V(x)=0, y \in Y(x)$ where $V(x):=\inf _{y \in Y(x)} f(x, y)$ is the value function of the lower level program for a numerical consideration. This so-called value function approach was further used in Ye and Zhu [32] and later in other papers such as [7, 8, 21, 22, 23, 24] to derive various necessary optimality conditions under the partial calmness condition. The partial calmness condition for the value function reformulation of the bilevel program, however, is a very strong assumption. To derive a necessary optimality condition under weaker assumptions, Ye and Zhu [33] proposed the following combined program where both the value function constraint and the KKT condition of the lower level program are used as constraints:

$$
\begin{array}{rl}
(\mathrm{CP}) \quad \min _{x, y, u, v} & F(x, y) \\
\text { s.t. } \quad & f(x, y)-V(x) \leq 0, G(x, y) \leq 0, H(x, y)=0, \\
& \nabla_{y} f(x, y)+\nabla_{y} g(x, y)^{T} u+\nabla_{y} h(x, y)^{T} v=0, \\
& (-g(x, y), u) \in \Omega^{m} .
\end{array}
$$

As discussed in [33], such a reformulation can avoid some difficulties caused by using the value function or the classical KKT approach alone. In [30, necessary optimality conditions in the form of Mordukhovich (M-) stationary condition for (CP) is studied under the partial calmness/weak calmness condition. These conditions, however, may not be easy to verify.

Note that the inclusion of the value function makes the problem nonsmooth since the value function is usually nonsmooth but under some reasonable assumptions on the lower level problem, the value function is Lipschitz continuous. Hence the feasible set of $(\mathrm{CP})$ is a special case of the general feasibility system (1.1). However as it was shown in Ye and Zhu [33, Proposition 1.3], the no nonzero abnormal multiplier constraint qualification (NNAMCQ) as defined in Definition 4.1 never holds for (CP). Being able to deal with nonsmooth inequality constraints in RCPLD would allow us to present verifiable constraint qualifications and exact penalty for the reformulation of the bilevel program in which the value function is used, such as (CP).

The rest of the paper is organized as follows. In Section 2, we present basic definitions as well as some preliminaries which will be used in this paper. In Section 3, we show that RCPLD is a constraint qualification and a sufficient condition for error bound properties under certain regularity conditions. We introduce some sufficient conditions for RCPLD, which are easier to verify in Section 4. In Section 5, we apply RCPLD to the bilevel program.

We adopt the following standard notation in this paper. For any two vectors $a$ and $b$, we denote by either $\langle a, b\rangle$ or $a^{T} b$ their inner product. Given a differentiable function $G: \mathbb{R}^{d} \rightarrow \mathbb{R}$, 
we denote its gradient vector by $\nabla G(x) \in \mathbb{R}^{d}$. For a differentiable mapping $\Phi: \mathbb{R}^{d} \rightarrow \mathbb{R}^{n}$ with $n \geq 2$ and a vector $x \in \mathbb{R}^{d}$, we denote by $\nabla \Phi(x) \in \mathbb{R}^{n \times d}$ the Jacobian matrix of $\Phi$ at $x$. For a set $C \subseteq \mathbb{R}^{d}$, we denote by int $C$, co $C, \bar{C}$, bd $C$ and $d_{C}(x)$ the interior, the convex hull, the closure, the boundary of $C$ and the distance from $x$ to $C$, respectively. We denote by $|\mathcal{I}|$ the cardinality of index set $\mathcal{I} \subseteq\{1, \ldots, n\}$. For any vector $v \in \mathbb{R}^{n}$ and a given index set $\mathcal{I} \subseteq\{1, \ldots, n\}$, we use $v_{\mathcal{I}}$ to denote the vector in $\mathbb{R}^{|\mathcal{I}|}$ with components $v_{i}, i \in \mathcal{I}$. For a matrix $A \in \mathbb{R}^{n \times m}, A^{T}$ denotes its transpose, $r(A)$ denotes its rank. We denote by $\mathbb{B}_{\delta}(\bar{x})$ the open ball center at $\bar{x}$ with radius $\delta>0$ and by $\mathbb{B}$ the open unit ball center at the origin. Unless otherwise specified we denote by $\|\cdot\|$ any norm in the finite dimensional space.

\section{Background and preliminaries}

In this section, we present some background materials on variational analysis which will be used throughout the paper. Detailed discussions on these subjects can be found in [4, 5, 21, 27].

Given a set $C \subseteq \mathbb{R}^{d}$, its Fréchet/regular normal cone at $z \in C$ is defined by

$$
\widehat{\mathcal{N}}_{C}(z):=\left\{v \in \mathbb{R}^{d}:\left\langle v, z^{\prime}-z\right\rangle \leq o\left(\left\|z-z^{\prime}\right\|\right) \text { for all } z^{\prime} \in C\right\}
$$

and the limiting/Mordukhovich/basic normal cone to $C$ at point $z$ is defined by

$$
\mathcal{N}_{C}(z)=\left\{\lim _{k \rightarrow \infty} v_{k}: v_{k} \in \widehat{\mathcal{N}}_{C}\left(z_{k}\right), \quad z_{k} \in C, z_{k} \rightarrow z\right\}
$$

A set $C \subseteq \mathbb{R}^{d}$ is Clarke regular at $z$ if it is locally closed at $z$ and $\mathcal{N}_{C}(z)=\widehat{\mathcal{N}}_{C}(z)$ [27, Definition 6.4]. Note that for $C:=C_{1} \times C_{2} \times \cdots \times C_{l}$ with $C_{i}$ closed, $i=1, \cdots, l$, by [27, Proposition 6.41], $C$ is regular at $z$ if and only if each $C_{i}$ is regular at $z_{i}$.

The exact expressions for the limiting normal cone of the complementarity set present below will be useful. In this paper we denote by $\Omega^{p}$ the complementarity set and when $p=1$, $\Omega:=\Omega^{1}=\left\{(y, z) \in \mathbb{R}^{2} \mid 0 \leq y \perp z \geq 0\right\}$.

Proposition 2.1 (See e.g. [29, Proposition 3.7]) For any (a,b) lying in the complementarity set $\Omega^{p}$, the limiting normal cone to $\Omega^{p}$ at $(a, b)$ is

$$
\mathcal{N}_{\Omega^{p}}(a, b)=\left\{\begin{array}{ll}
\alpha_{i} \in \mathbb{R}, \beta_{i}=0 & \text { if } a_{i}=0<b_{i} \\
-(\alpha, \beta) \in \mathbb{R}^{p} \times \mathbb{R}^{p}: & \alpha_{i}=0, \beta_{i} \in \mathbb{R} \\
& \text { if } a_{i}>0=b_{i} \\
\text { either } \alpha_{i}>0, \beta_{i}>0 \text { or } \alpha_{i} \beta_{i}=0 & \text { if } a_{i}=b_{i}=0
\end{array}\right\} .
$$

Let $f: \mathbb{R}^{d} \rightarrow \overline{\mathbb{R}}$ be a lower semicontinuous function and finite at $x \in \mathbb{R}^{d}$. We define the Fréchet/regular subdifferential ([27, Definition 8.3]) of $f$ at $x$ as

$$
\widehat{\partial} f(x):=\left\{\zeta \in \mathbb{R}^{d}: f\left(x^{\prime}\right)-f(x)-\left\langle\zeta, x^{\prime}-x\right\rangle \geq o\left\|x^{\prime}-x\right\|\right\}
$$


and the limiting/Mordukhovich/basic subdifferential of $f$ at $x$ as

$$
\partial f(x):=\left\{\lim _{k \rightarrow \infty} \xi_{k}: \xi_{k} \in \widehat{\partial} f\left(x_{k}\right), x_{k} \rightarrow x, f\left(x_{k}\right) \rightarrow f(x)\right\} .
$$

Let $f: \mathbb{R}^{d} \rightarrow \mathbb{R}$ be Lipschitz continuous at $x$. We say that $f$ is subdifferentially/Clarke regular at $x$ provided that $\partial f(x)=\widehat{\partial} f(x)$ [27, Corollary 8.11].

The following proposition collects some useful properties and calculus rules of the limiting subdifferential.

Proposition 2.2 (i) [27, Exercise 10.10] and [21, Theorem 2.33] Let $f, g: \mathbb{R}^{n} \rightarrow[-\infty, \infty]$ be proper lower semi-continuous around $x^{*} \in \mathbb{R}^{n}$ and finite at $x^{*}$, and $\alpha, \beta$ be nonnegative scalars. Assume that at least one of them is Lipschitz continuous around $x^{*}$. Then

$$
\partial(\alpha f+\beta g)\left(x^{*}\right) \subseteq \alpha \partial f\left(x^{*}\right)+\beta \partial g\left(x^{*}\right)
$$

(ii) [17, Theorem 2.5, Remark (2)] and [21, Theorem 3.41]Let $g: \mathbb{R}^{n} \rightarrow \mathbb{R}^{m}$ be Lipschitz continuous around $x^{*}$ and $f: \mathbb{R}^{m} \rightarrow \mathbb{R}$ be Lipschitz continuous near $g\left(x^{*}\right)$. Then the composite function $f \circ g$ is Lipschitz continuous around $x^{*}$ and

$$
\partial(f \circ g)\left(x^{*}\right) \subseteq \bigcup_{\xi \in \partial f\left(g\left(x^{*}\right)\right)} \partial\langle\xi, g\rangle\left(x^{*}\right) .
$$

(iii) [21, Theorem 3.46 and Proposition 1.113] Let $\varphi_{i}: \mathbb{R}^{n} \rightarrow \mathbb{R}(i=1, \ldots, n)$ be Lipschitz continuous around $x^{*}$ and $\varphi_{\max }(x):=\max \left\{\varphi_{i}(x): i=1, \ldots, n\right\}$, and $\varphi_{\min }(x):=\min \left\{\varphi_{i}(x):\right.$ $i=1, \ldots, n\}$. Then $\varphi_{\max }(x)$ and $\varphi_{\min }(x)$ are Lipschitz continuous around $x^{*} \in \mathbb{R}^{n}$ and

$$
\begin{aligned}
\partial \varphi_{\max }\left(x^{*}\right) & \subseteq \operatorname{co}\left\{\partial \varphi_{i}\left(x^{*}\right): i \in I_{+}\left(x^{*}\right)\right\} \\
\partial \varphi_{\min }\left(x^{*}\right) & \subseteq\left\{\partial \varphi_{i}\left(x^{*}\right): i \in I_{-}\left(x^{*}\right)\right\}
\end{aligned}
$$

where $I_{+}\left(x^{*}\right):=\left\{i: \varphi_{i}\left(x^{*}\right)=\varphi_{\max }\left(x^{*}\right)\right\}$ and $I_{-}\left(x^{*}\right):=\left\{i: \varphi_{i}\left(x^{*}\right)=\varphi_{\min }\left(x^{*}\right)\right\}$, and the first inclusion holds as an equation if each $\varphi_{i}$ is subdifferentially regular at $x^{*}$.

Taking into account that all norms in a finite dimensional space are equivalent, the following formula for distance function to the complementarity set $\Omega$ can be used in the error bound property (1.3).

Proposition 2.3 (see e.g. [18]) When the norm is chosen to be the $l_{1}$-norm in the distance function $d_{\Omega}$, for any $(a, b) \in \mathbb{R}^{2}$,

$$
d_{\Omega}(a, b)=\max \{-a,-b,-(a+b), \min \{a, b\}\}
$$

When the norm is chosen to be the $l_{\infty}$-norm in the distance function $d_{\Omega}$, for any $(a, b) \in \mathbb{R}^{2}$,

$$
d_{\Omega}(a, b)=|\min \{a, b\}|
$$


In Theorem 3.1, we need to calculate $\partial \phi_{0}(x)$, where

$$
\phi_{0}(x):=\sum_{i=1}^{n} \max \left\{0, g_{i}(x)\right\}+\sum_{i=1}^{m}\left|h_{i}(x)\right|+\sum_{i=1}^{p}\left|\min \left\{G_{i}(x), H_{i}(x)\right\}\right| .
$$

In order to calculate $\partial \phi_{0}(x)$, we define the following index sets for each $x$ :

$$
\begin{aligned}
& A(x):=\left\{i=1, \cdots, n: g_{i}(x) \geq 0\right\}, \\
& \mathcal{I}(x):=\left\{i=1, \cdots, p: G_{i}(x)<H_{i}(x)\right\}, \\
& \mathcal{J}(x):=\left\{i=1, \cdots, p: G_{i}(x)=H_{i}(x)\right\}, \\
& \mathcal{K}(x):=\left\{i=1, \cdots, p: G_{i}(x)>H_{i}(x)\right\} .
\end{aligned}
$$

From the calculus rules in Proposition 2.2, we have the following estimate for the limiting subdifferential of $\phi_{0}$.

Lemma 2.1 Assume that the functions $g_{i}: \mathbb{R}^{d} \rightarrow \mathbb{R}, i=1, \cdots, n$, are locally Lipschitz continuous and $h_{i}: \mathbb{R}^{d} \rightarrow \mathbb{R}, i=1, \cdots, m, G, H: \mathbb{R}^{d} \rightarrow \mathbb{R}^{p}$, are continuously differentiable around $x^{*} . \phi_{0}(x)$ is a Lipschitz continuous function and for any $x^{*}$, there exist $\lambda_{i}^{g} \geq 0, i \in A\left(x^{*}\right)$, $\lambda_{i}^{h}, i=1, \cdots, m$ and $\lambda_{i}^{G}, \lambda_{i}^{H}, i=1, \cdots, p$ satisfying

$$
\begin{cases}\lambda_{i}^{H}=0 & \text { if } i \in \mathcal{I}\left(x^{*}\right) \\ \lambda_{i}^{G}=0 & \text { if } i \in \mathcal{K}\left(x^{*}\right) \\ \text { either } \lambda_{i}^{G}>0, \lambda_{i}^{H}>0 \text { or } \lambda_{i}^{G} \lambda_{i}^{H}=0 & \text { if } i \in \mathcal{J}\left(x^{*}\right)\end{cases}
$$

such that

$$
\partial \phi_{0}\left(x^{*}\right) \subseteq \sum_{i \in A\left(x^{*}\right)} \lambda_{i}^{g} \partial g_{i}\left(x^{*}\right)+\sum_{i=1}^{m} \lambda_{i}^{h} \nabla h_{i}\left(x^{*}\right)-\sum_{i=1}^{p} \lambda_{i}^{G} \nabla G_{i}\left(x^{*}\right)-\sum_{i=1}^{p} \lambda_{i}^{H} \nabla H_{i}\left(x^{*}\right) .
$$

Proof. For $i=1, \cdots, p$, let $F_{i}(x):=\left|f_{i}(x)\right|$ and $f_{i}(x):=\min \left\{G_{i}(x), H_{i}(x)\right\}$. From the chain rule in Proposition $2.2\left(\right.$ ii), we have $\partial F_{i}\left(x^{*}\right) \subseteq\left\{\partial\left(\mu f_{i}\right)\left(x^{*}\right): \mu \in \partial|\cdot|\left(f_{i}\left(x^{*}\right)\right)\right\}$. We divide the analysis into three cases.

Case 1: $i \in \mathcal{I}\left(x^{*}\right)$. In this case we have $G_{i}\left(x^{*}\right)<H_{i}\left(x^{*}\right)$ and hence $f_{i}\left(x^{*}\right)=G_{i}\left(x^{*}\right)$. By the chain rule we have $\partial F_{i}(x)=\left\{\nabla G_{i}\left(x^{*}\right)\right\}$ if $G_{i}\left(x^{*}\right)>0$ and $\partial F_{i}(x)=\left\{-\nabla G_{i}\left(x^{*}\right)\right\}$ if $G_{i}\left(x^{*}\right)<0$. If $G_{i}\left(x^{*}\right)=0$, then $\partial|\cdot|\left(f_{i}\left(x^{*}\right)\right)=[-1,1]$ and hence $\partial F_{i}(x) \subseteq\left\{\mu \nabla G_{i}\left(x^{*}\right): \mu \in[-1,1]\right\}$.

Case 2: $i \in \mathcal{K}\left(x^{*}\right)$. Similarly as in Case 1 , we can show $\partial F_{i}(x) \subseteq\left\{\mu \nabla H_{i}\left(x^{*}\right): \mu \in[-1,1]\right\}$.

Case 3: $i \in \mathcal{J}\left(x^{*}\right)$. In this case, $f_{i}\left(x^{*}\right)=G_{i}\left(x^{*}\right)=H_{i}\left(x^{*}\right)$. If $G_{i}\left(x^{*}\right)=H_{i}\left(x^{*}\right)<0$, then $\partial|\cdot|\left(f_{i}\left(x^{*}\right)\right)=\{-1\}$ and $\partial F_{i}\left(x^{*}\right) \subseteq \partial\left(-f_{i}\right)\left(x^{*}\right)$. Since $-f_{i}(x)=\max \left\{-G_{i}(x),-H_{i}(x)\right\}$, by Proposition 2.2(iii), $\partial F_{i}(x)=c o\left\{-\nabla G_{i}\left(x^{*}\right),-\nabla H_{i}\left(x^{*}\right)\right\}$. If $G_{i}\left(x^{*}\right)=H_{i}\left(x^{*}\right)>0$, then $\partial|\cdot|\left(f_{i}\left(x^{*}\right)\right)=\{1\}$ and $\partial F_{i}\left(x^{*}\right) \subseteq \partial f_{i}\left(x^{*}\right)$. It follows by Proposition 2.2(iii) that $\partial F_{i}(x) \subseteq$ 
$\left\{\nabla G_{i}\left(x^{*}\right), \nabla H_{i}\left(x^{*}\right)\right\}$. If $G_{i}\left(x^{*}\right)=H_{i}\left(x^{*}\right)=0$, then $\partial|\cdot|\left(f_{i}\left(x^{*}\right)\right)=[-1,1], \partial F_{i}(x) \subseteq$ $\left\{\partial\left(\mu f_{i}\right)\left(x^{*}\right): \mu \in[-1,1]\right\}$. If $\mu>0$, then $\partial\left(\mu f_{i}\right)\left(x^{*}\right) \subseteq \mu \partial f_{i}\left(x^{*}\right) \subseteq \mu\left\{\nabla G_{i}\left(x^{*}\right), \nabla H_{i}\left(x^{*}\right)\right\}$. If $\mu<0$, then $\partial\left(\mu f_{i}\right)\left(x^{*}\right)=-\mu \partial\left(-f_{i}\right)\left(x^{*}\right)=-\mu c o\left\{-\nabla G_{i}\left(x^{*}\right),-\nabla H_{i}\left(x^{*}\right)\right\}$. Hence

$$
\partial F_{i}\left(x^{*}\right) \subseteq\left\{\lambda v: \lambda \geq 0, v \in c o\left\{-\nabla G_{i}\left(x^{*}\right),-\nabla H_{i}\left(x^{*}\right)\right\} \cup\left\{\nabla G_{i}\left(x^{*}\right), \nabla H_{i}\left(x^{*}\right)\right\}\right\} .
$$

Summarizing the above cases, we have that for any $x^{*}$,

$$
\partial\left|\min \left\{G_{i}\left(x^{*}\right), H_{i}\left(x^{*}\right)\right\}\right| \subseteq\left(\begin{array}{c}
\nabla G\left(x^{*}\right) \\
\nabla H\left(x^{*}\right)
\end{array}\right)^{T}\left(-\lambda^{G},-\lambda^{H}\right),
$$

where (2.2) holds.

Since $g_{i}(x), i=1, \cdots, n,\left|h_{i}(x)\right|, j=1, \cdots, m$ and $\left|\min \left\{G_{i}(x), H_{i}(x)\right\}\right|, i=1 \cdots, p$ are all Lipschitz continuous around $x^{*}$, by the calculus rules in Proposition 2.2 and (2.2), there exist parameters $\lambda_{i}^{g} \geq 0, i \in A\left(x^{*}\right), \lambda_{i}^{h}, i=1, \cdots, m$ and $\lambda_{i}^{G}, \lambda_{i}^{H}, i=1, \cdots, p$ satisfying (2.2) such that

$$
\partial \phi_{0}\left(x^{*}\right) \subseteq \sum_{i \in A\left(x^{*}\right)} \lambda_{i}^{g} \partial g_{i}\left(x^{*}\right)+\sum_{i=1}^{m} \lambda_{i}^{h} \nabla h_{i}\left(x^{*}\right)-\sum_{i=1}^{p} \lambda_{i}^{G} \nabla G_{i}\left(x^{*}\right)-\sum_{i=1}^{p} \lambda_{i}^{H} \nabla H_{i}\left(x^{*}\right) .
$$

We will need the following result which is an extension of Carathéodory's lemma.

Lemma 2.2 [1, Lemma 1] If $v=\sum_{i=1}^{m+n} \alpha_{i} v_{i}$ with $v_{i} \in \mathbb{R}^{d}$ for every $i,\left\{v_{i}\right\}_{i=1}^{m}$ is linearly independent and $\alpha_{i} \neq 0, i=m+1, \cdots, m+n$, then there exist $I \subseteq\{m+1, \cdots, m+n\}$ and scalars $\bar{\alpha}_{i}$ for every $i \in\{1, \cdots, m\} \cup I$ such that

(i) $v=\sum_{\{1, \cdots, m\} \cup I} \bar{\alpha}_{i} v_{i}$ with $\alpha_{i} \bar{\alpha}_{i}>0$ for every $i \in I$,

(ii) $\left\{v_{i}\right\}_{\{1, \cdots, m\} \cup I}$ is linearly independent.

\section{RCPLD as a constraint qualification and a sufficient condition for error bounds}

We first show that the RCPLD introduced in Definition 1.1 is a constraint qualification for any optimization problem in the form (P) $\min _{x} \quad f(x)$ s.t. $\quad x \in \mathcal{F}$, where $f: \mathbb{R}^{d} \rightarrow \mathbb{R}$ is Lipschitz continuous around any local optimal solution and $\mathcal{F}$ is the feasible region defined by the system (1.1).

Theorem 3.1 Let $x^{*}$ be a local minimizer of the optimization problem (P). Suppose that RCPLD holds at $x^{*}$. Then $x^{*}$ is an M-stationary point, i.e., there exist $\lambda_{i}^{g} \geq 0, i \in I_{g}^{*}, \lambda_{i}^{h}$, 
$i=1, \cdots, m, \lambda_{i}^{G}, i=1, \cdots, p$ and $\lambda_{i}^{H}, i=1, \cdots, p$ such that

$$
\begin{aligned}
0 \in & \partial f\left(x^{*}\right)+\sum_{i \in I_{g}^{*}} \lambda_{i}^{g} \partial g_{i}\left(x^{*}\right)+\sum_{i=1}^{m} \lambda_{i}^{h} \nabla h_{i}\left(x^{*}\right) \\
& -\sum_{i \in \mathcal{I}^{*} \cup \mathcal{J}^{*}} \lambda_{i}^{G} \nabla G_{i}\left(x^{*}\right)-\sum_{i \in \mathcal{K}^{*} \cup \mathcal{J}^{*}} \lambda_{i}^{H} \nabla H_{i}\left(x^{*}\right)+\mathcal{N}_{C}\left(x^{*}\right), \\
\lambda_{i}^{H} & =0 i \in \mathcal{I}^{*}, \lambda_{i}^{G}=0 i \in \mathcal{K}^{*}, \text { either } \lambda_{i}^{G}>0, \lambda_{i}^{H}>0 \text {, or } \lambda_{i}^{G} \lambda_{i}^{H}=0, i \in \mathcal{J}^{*} .
\end{aligned}
$$

Proof. Step 1: Note that a point $x \in C$ is feasible for problem $(\mathrm{P})$ if and only if

$$
\phi_{0}(x):=\sum_{i=1}^{n} \max \left\{0, g_{i}(x)\right\}+\sum_{i=1}^{m}\left|h_{i}(x)\right|+\sum_{i=1}^{p}\left|\min \left\{G_{i}(x), H_{i}(x)\right\}\right|=0 .
$$

For each $k$, we consider the following penalized problem:

$$
\begin{aligned}
\left(\mathrm{P}_{k}\right) \quad \min & F^{k}(x):=f(x)+\frac{k}{2} \phi_{0}^{2}(x)+\frac{1}{2}\left\|x-x^{*}\right\|^{2} \\
\text { s.t. } & x \in \overline{\mathbb{B}}_{\varepsilon}\left(x^{*}\right) \cap C,
\end{aligned}
$$

where $\|\cdot\|$ denotes the 2-norm, $\varepsilon>0$ is such that $f\left(x^{*}\right) \leq f(x)$ for all feasible point $x \in \overline{\mathbb{B}}_{\varepsilon}\left(x^{*}\right)$. Since the feasible region is compact and the objective function is continuous, there exists an optimal solution $x^{k}$ of the problem $\left(\mathrm{P}_{k}\right)$. Taking subsequence if necessary, we assume that $\lim _{k \rightarrow \infty} x^{k}=\bar{x}$ and thus $\bar{x} \in \overline{\mathbb{B}}_{\varepsilon}\left(x^{*}\right) \cap C$. Moreover by the optimality of $x^{k}$, we have

$$
f\left(x^{k}\right)+\frac{k}{2} \phi_{0}^{2}\left(x^{k}\right)+\frac{1}{2}\left\|x^{k}-x^{*}\right\|^{2}=F^{k}\left(x^{k}\right) \leq F^{k}\left(x^{*}\right)=f\left(x^{*}\right) .
$$

From the boundedness of $\left\{f\left(x^{k}\right)\right\}$, we have that $\phi_{0}\left(x^{k}\right) \rightarrow 0$ as $k \rightarrow \infty$. It follows that $\bar{x}$ is a feasible point of the problem $(P)$. Condition (3.1) yields $f\left(x^{k}\right)+\frac{1}{2}\left\|x^{k}-x^{*}\right\|^{2} \leq f\left(x^{*}\right)$. Taking limit as $k \rightarrow \infty$, we obtain $f(\bar{x})+\frac{1}{2}\left\|x^{*}-\bar{x}\right\|^{2} \leq f\left(x^{*}\right)$, which means that $\bar{x}=x^{*}$. Thus the sequence $\left\{x^{k}\right\}$ converges to $x^{*}$.

Step 2: Since for sufficiently large $k, x^{k}$ is an interior point of $\overline{\mathbb{B}}_{\varepsilon}\left(x^{*}\right)$, by the necessary optimality condition in terms of limiting subdifferential and the nonsmooth calculus rule, there exist $v_{0}^{k} \in \partial f\left(x^{k}\right), u^{k} \in k \phi_{0}\left(x^{k}\right) \partial \phi_{0}\left(x^{k}\right)+\eta^{k}, \eta^{k} \in \mathcal{N}_{C}\left(x^{k}\right)$ such that

$$
0=v_{0}^{k}+u^{k}+\left(x^{k}-x^{*}\right)
$$

Suppose that there is a subsequence of $\left\{u^{k}\right\}$ such that all $u^{k}$ equal to zero in this subsequence. Since $f$ is Lipschitz continuous, its limiting subdifferential is compact and so the sequences $\left\{v_{0}^{k}\right\}$ is compact. Taking subsequence if necessary, we assume $\lim _{k \rightarrow 0} u^{k}=0$ and $\lim _{k \rightarrow \infty} v_{0}^{k}=v_{0} \in \partial f\left(x^{*}\right)$. Taking limit as $k \rightarrow \infty$ in (3.2), we have $0 \in \partial f\left(x^{*}\right)$ by the outer semi-continuity of the limiting subdifferential and thus $x^{*}$ is a stationary point of problem $(\mathrm{P})$ automatically. So without loss of generality, we may assume $u^{k} \neq 0$ for all sufficiently large $k$. 
By Lemma 2.1, since $k \phi_{0}\left(x^{k}\right) \geq 0$ there exist $\lambda_{i, k}^{g} \geq 0, i \in A\left(x^{k}\right), \lambda_{i, k}^{h}, i=1, \cdots, m, v_{i}^{k} \in$ $\partial g_{i}\left(x^{k}\right), i \in A\left(x^{k}\right)$ and $\lambda_{i, k}^{G}, \lambda_{i, k}^{H}, i=1, \cdots, p$ such that either $\lambda_{i, k}^{G}>0, \lambda_{i, k}^{H}>0$ or $\lambda_{i, k}^{G} \lambda_{i, k}^{H}=$ $0, \quad \forall i \in \mathcal{J}\left(x^{k}\right)$ and

$$
\begin{aligned}
& k \phi_{0}\left(x^{k}\right) \partial \phi_{0}\left(x^{k}\right) \subseteq \\
& \sum_{i \in A\left(x^{k}\right)} \lambda_{i, k}^{g} v_{i}^{k}+\sum_{i=1}^{m} \lambda_{i, k}^{h} \nabla h_{i}\left(x^{k}\right)-\sum_{i \in \mathcal{I}\left(x^{k}\right) \cup \mathcal{J}\left(x^{k}\right)} \lambda_{i, k}^{G} \nabla G_{i}\left(x^{k}\right)-\sum_{i \in \mathcal{K}\left(x^{k}\right) \cup \mathcal{J}\left(x^{k}\right)} \lambda_{i, k}^{H} \nabla H_{i}\left(x^{k}\right) .
\end{aligned}
$$

By the continuity of $g$, it is easy to see that $A\left(x^{k}\right) \subseteq I_{g}^{*}$ for sufficiently large $k$. Similarly, by the continuity of $G$ and $H, \mathcal{I}\left(x^{k}\right) \cup \mathcal{J}\left(x^{k}\right) \subseteq \mathcal{I}^{*} \cup \mathcal{J}^{*}$ and $\mathcal{K}\left(x^{k}\right) \cup \mathcal{J}\left(x^{k}\right) \subseteq \mathcal{K}^{*} \cup \mathcal{J}^{*}$ for sufficiently large $k$. Let $\lambda_{i, k}^{g}=0$ if $i \in I_{g}^{*} \backslash A\left(x^{k}\right), \lambda_{i, k}^{G}=0$ if $i \in\left(\mathcal{I}^{*} \cup \mathcal{J}^{*}\right) \backslash\left(\mathcal{I}\left(x^{k}\right) \cup \mathcal{J}\left(x^{k}\right)\right)$, and $\lambda_{i, k}^{H}=0$ if $i \in\left(\mathcal{K}^{*} \cup \mathcal{J}^{*}\right) \backslash\left(\mathcal{K}\left(x^{k}\right) \cup \mathcal{J}\left(x^{k}\right)\right)$. Then we have that $\lambda_{i, k}^{G}>0, \lambda_{i, k}^{H}>0$ or $\lambda_{i, k}^{G} \lambda_{i, k}^{H}=0, \quad \forall i \in \mathcal{J}^{*}$. Since $u^{k} \in k \phi_{0}\left(x^{k}\right) \partial \phi_{0}\left(x^{k}\right)+\eta^{k}$, it follows that

$$
u^{k}=\sum_{i \in I_{g}^{*}} \lambda_{i, k}^{g} v_{i}^{k}+\sum_{i=1}^{m} \lambda_{i, k}^{h} \nabla h_{i}\left(x^{k}\right)-\sum_{i \in \mathcal{I}^{*} \cup \mathcal{J}^{*}} \lambda_{i, k}^{G} \nabla G_{i}\left(x^{k}\right)-\sum_{i \in \mathcal{K}^{*} \cup \mathcal{J}^{*}} \lambda_{i, k}^{H} \nabla H_{i}\left(x^{k}\right)+\eta^{k} .
$$

Since $\eta^{k}:=\left(\eta_{1}^{k}, \ldots, \eta_{l}^{k}\right) \in \mathcal{N}_{C}\left(x^{k}\right)=\mathcal{N}_{C_{1}}\left(x_{1}^{k}\right) \times \cdots \times \mathcal{N}_{C_{l}}\left(x_{l}^{k}\right)$, we have $\nu_{i}^{k}:=\{0\}^{s_{i}} \times\left\{\eta_{i}^{k}\right\} \times\{0\}^{t_{i}} \in$ $\mathcal{N}_{C}\left(x^{k}\right)$. We denote by $\eta^{k}=\sum_{i \in L_{k}} \nu_{i}^{k}$ with $L_{k}:=\left\{i=1, \cdots, l: \eta_{i}^{k} \neq 0\right\}$.

Let $\mathcal{I}_{1} \subseteq\{1, \cdots, m\}, \mathcal{I}_{2} \in \mathcal{I}^{*}$ and $\mathcal{I}_{3} \in \mathcal{K}^{*}$ be such that $\left\{\nabla h_{i}\left(x^{*}\right)\right\}_{i \in \mathcal{I}_{1}} \cup\left\{\nabla G_{i}\left(x^{*}\right)\right\}_{i \in \mathcal{I}_{2}} \cup$ $\left\{\nabla H_{i}\left(x^{*}\right)\right\}_{i \in \mathcal{I}_{3}}$ is a basis for $\operatorname{span}\left\{\left\{\nabla h_{i}\left(x^{*}\right)\right\}_{i=1}^{m} \cup\left\{\nabla G_{i}\left(x^{*}\right)\right\}_{i \in \mathcal{I}^{*}} \cup\left\{\nabla H_{i}\left(x^{*}\right)\right\}_{i \in \mathcal{K}^{*}}\right\}$. Then by $\operatorname{RCPLD}(\mathrm{i}),\left\{\nabla h_{i}\left(x^{k}\right)\right\}_{i \in \mathcal{I}_{1}} \cup\left\{\nabla G_{i}\left(x^{k}\right)\right\}_{i \in \mathcal{I}_{2}} \cup\left\{\nabla H_{i}\left(x^{k}\right)\right\}_{i \in \mathcal{I}_{3}}$ is linearly independent and thus is a basis of span $\left\{\left\{\nabla h_{i}\left(x^{k}\right)\right\}_{i=1}^{m} \cup\left\{\nabla G_{i}\left(x^{k}\right)\right\}_{i \in \mathcal{I}^{*}} \cup\left\{\nabla H_{i}\left(x^{k}\right)\right\}_{i \in \mathcal{K}^{*}}\right\}$. Hence there exist $\tilde{\lambda}_{i, k}^{h}, i \in \mathcal{I}_{1}$, $\tilde{\lambda}_{k}^{G}, \tilde{\lambda}_{k}^{H}$ such that

$$
\begin{array}{r}
u^{k}=\sum_{i \in I_{g}^{*} \cap \operatorname{supp}\left(\lambda_{i, k}^{g}\right)} \lambda_{i, k}^{g} v_{i}^{k}+\sum_{i \in \mathcal{I}_{1}} \tilde{\lambda}_{i, k}^{h} \nabla h_{i}\left(x^{k}\right)-\sum_{i \in \mathcal{I}_{2}} \tilde{\lambda}_{i, k}^{G} \nabla G_{i}\left(x^{k}\right)-\sum_{i \in \mathcal{I}_{3}} \tilde{\lambda}_{i, k}^{H} \nabla H_{i}\left(x^{k}\right) \\
-\sum_{i \in \mathcal{J}^{*} \cap \operatorname{supp}\left(\tilde{\lambda}_{i, k}^{G}\right)} \tilde{\lambda}_{i, k}^{G} \nabla G_{i}\left(x^{k}\right)-\sum_{i \in \mathcal{J}^{*} \cap \operatorname{supp}\left(\tilde{\lambda}_{i, k}^{H}\right)} \tilde{\lambda}_{i, k}^{H} \nabla H_{i}\left(x^{k}\right)+\sum_{i \in L_{k}} \nu_{i}^{k},
\end{array}
$$

where $\operatorname{supp}(a):=\left\{i: a_{i} \neq 0\right\}, \tilde{\lambda}_{i, k}^{G}>0, \tilde{\lambda}_{i, k}^{H}>0$ or $\tilde{\lambda}_{i, k}^{G} \tilde{\lambda}_{i, k}^{H}=0$ if $i \in \mathcal{J}^{*}$.

Since $u^{k} \neq 0$, applying Carathéodory's lemma in Lemma 2.2 to (3.3), we obtain subsets

$$
\mathcal{I}_{4}^{k} \subseteq I_{g}^{*} \cap \operatorname{supp}\left(\tilde{\lambda}_{i, k}^{g}\right), \quad \mathcal{I}_{5}^{k} \subseteq \mathcal{J}^{*} \cap \operatorname{supp}\left(\tilde{\lambda}_{i, k}^{G}\right), \quad \mathcal{I}_{6}^{k} \subseteq \mathcal{J}^{*} \cap \operatorname{supp}\left(\tilde{\lambda}_{i, k}^{H}\right), \quad L_{k}^{\prime} \subseteq L_{k},
$$

and $\left\{\bar{\lambda}_{k}^{g}, \bar{\lambda}_{k}^{h}, \bar{\lambda}_{k}^{G}, \bar{\lambda}_{k}^{H}, \alpha^{k}\right\}$ with $\alpha_{i}^{k}>0, i \in L_{k}^{\prime}, \bar{\lambda}_{i, k}^{g}>0, i \in \mathcal{I}_{4}^{k}$ and $\bar{\lambda}_{i, k}^{G}>0, \bar{\lambda}_{i, k}^{H}>0$ or $\bar{\lambda}_{i, k}^{G} \bar{\lambda}_{i, k}^{H}=0$ if $i \in \mathcal{J}^{*}$ such that

$$
u^{k}=\sum_{i \in \mathcal{I}_{4}^{k}} \bar{\lambda}_{i, k}^{g} v_{i}^{k}+\sum_{i \in \mathcal{I}_{1}} \bar{\lambda}_{i, k}^{h} \nabla h_{i}\left(x^{k}\right)-\sum_{i \in \mathcal{I}_{2} \cup \mathcal{I}_{5}^{k}} \bar{\lambda}_{i, k}^{G} \nabla G_{i}\left(x^{k}\right)-\sum_{i \in \mathcal{I}_{3} \cup \mathcal{I}_{6}^{k}} \bar{\lambda}_{i, k}^{H} \nabla H_{i}\left(x^{k}\right)+\sum_{i \in L_{k}^{\prime}} \alpha_{i}^{k} \nu_{i}^{k},(3.4)
$$

and the set of vectors $\left\{v_{i}^{k}\right\}_{i \in \mathcal{I}_{4}^{k}} \cup\left\{\nabla h_{i}\left(x^{k}\right)\right\}_{i \in \mathcal{I}_{1}} \cup\left\{\nabla G_{i}\left(x^{k}\right)\right\}_{i \in \mathcal{I}_{2} \cup \mathcal{I}_{5}^{k}} \cup\left\{\nabla H_{i}\left(x^{k}\right)\right\}_{i \in \mathcal{I}_{3} \cup \mathcal{I}_{6}^{k}} \cup\left\{\nu_{i}^{k}\right\}_{i \in L_{k}^{\prime}}$ is linearly independent. 
Since the index sets are all finite, for every large $k$, we may assume $\mathcal{I}_{4}^{k} \equiv \mathcal{I}_{4}, L_{k}^{\prime} \equiv L, \mathcal{I}_{5}^{k} \equiv \mathcal{I}_{5}$ and $\mathcal{I}_{6}^{k} \equiv \mathcal{I}_{6}$ without loss of generality. Hence the set of vectors $\left\{v_{i}^{k}\right\}_{i \in \mathcal{I}_{4}} \cup\left\{\nabla h_{i}\left(x^{k}\right)\right\}_{i \in \mathcal{I}_{1}} \cup$ $\left\{\nabla G_{i}\left(x^{k}\right)\right\}_{i \in \mathcal{I}_{2} \cup \mathcal{I}_{5}} \cup\left\{\nabla H_{i}\left(x^{k}\right)\right\}_{i \in \mathcal{I}_{3} \cup \mathcal{I}_{6}} \cup\left\{\nu_{i}^{k}\right\}_{i \in L}$ is linearly independent. From (3.4), the condition (3.2) reduces to

$$
\begin{aligned}
0= & v_{0}^{k}+\left(x^{k}-x^{*}\right)+\sum_{i \in \mathcal{I}_{4}} \bar{\lambda}_{i, k}^{g} v_{i}^{k}+\sum_{i \in \mathcal{I}_{1}} \bar{\lambda}_{i, k}^{h} \nabla h_{i}\left(x^{k}\right)-\sum_{i \in \mathcal{I}_{2}} \bar{\lambda}_{i, k}^{G} \nabla G_{i}\left(x^{k}\right)-\sum_{i \in \mathcal{I}_{3}} \bar{\lambda}_{i, k}^{H} \nabla H_{i}\left(x^{k}\right) \\
& -\sum_{i \in \mathcal{I}_{5}} \bar{\lambda}_{i, k}^{G} \nabla G_{i}\left(x^{k}\right)-\sum_{i \in \mathcal{I}_{6}} \bar{\lambda}_{i, k}^{H} \nabla H_{i}\left(x^{k}\right)+\sum_{i \in L} \alpha_{i}^{k} \nu_{i}^{k}
\end{aligned}
$$

where $\alpha_{i}^{k}>0, i \in L, \bar{\lambda}_{i, k}^{g}>0$ if $i \in \mathcal{I}_{4}, \bar{\lambda}_{i, k}^{G}>0, \bar{\lambda}_{i, k}^{H}>0$ or $\bar{\lambda}_{i, k}^{G} \bar{\lambda}_{i, k}^{H}=0$ if $i \in \mathcal{J}^{*}$.

Step 3: We now prove that the sequence $\left\{\left(\bar{\lambda}_{k}^{g}, \bar{\lambda}_{k}^{h}, \bar{\lambda}_{k}^{G}, \bar{\lambda}_{k}^{H}, \sum_{i \in L} \alpha_{i}^{k} \nu_{i}^{k}\right)\right\}$ must be bounded. To the contrary, assume that it is unbounded. Let $M_{k}:=\left\|\left(\bar{\lambda}_{k}^{g}, \bar{\lambda}_{k}^{h}, \bar{\lambda}_{k}^{G}, \bar{\lambda}_{k}^{H}\right)\right\|+\left\|\sum_{i \in L} \alpha_{i}^{k} \nu_{i}^{k}\right\|$. Then there exists a subsequence $K$ such that $M_{k} \rightarrow \infty$ and

$$
\lim _{k \rightarrow \infty, k \in K} \frac{\left(\bar{\lambda}_{k}^{g}, \bar{\lambda}_{k}^{h}, \bar{\lambda}_{k}^{G}, \bar{\lambda}_{k}^{H}, \sum_{i \in L} \alpha_{i}^{k} \nu_{i}^{k}\right)}{M_{k}}=\left(\lambda^{g}, \lambda^{h}, \lambda^{G}, \lambda^{H}, \eta^{*}\right)
$$

where $\eta_{i}^{*}=\lim _{k \rightarrow \infty} \frac{\alpha_{i}^{k}}{M_{k}} \eta_{i}^{k}, i \in L$ and $\eta_{i}^{*}=0, i \notin L$. Since $\eta_{i}^{k} \in \mathcal{N}_{C_{i}}\left(x_{i}^{k}\right)$ and $\alpha_{i}^{k} / M_{k}>0$, it follows from the outer semicontinuity of the limiting normal cone that $\eta_{i}^{*} \in \mathcal{N}_{C_{i}}\left(x_{i}^{*}\right)$ and $\eta^{*} \in \mathcal{N}_{C}\left(x^{*}\right)$ for each $i \in L$. It is easy to see that $\lambda_{i}^{g} \geq 0$ if $i \in \mathcal{I}_{4}$ and either $\lambda_{i}^{G}>0, \lambda_{i}^{H}>$ 0 or $\lambda_{i}^{G} \lambda_{i}^{H}=0 \forall i \in \mathcal{J}^{*}$. Without loss of generality, assume that $v_{i}^{k} \rightarrow v_{i}^{*}$ and $v_{0}^{k} \rightarrow v_{0}^{*}$. Then by the outer semi-continuity of the limiting subdifferential, we have $v_{i}^{*} \in \partial g_{i}\left(x^{*}\right)$ and $v_{0}^{*} \in \partial f\left(x^{*}\right)$. Dividing $M_{k}$ on both sides of (3.5) and taking limits with $k \rightarrow \infty, k \in K$, we have

$$
0=\sum_{i \in \mathcal{I}_{4}} \lambda_{i}^{g} v_{i}^{*}+\sum_{i \in \mathcal{I}_{1}} \lambda_{i}^{h} \nabla h_{i}\left(x^{*}\right)-\sum_{i \in \mathcal{I}_{2} \cup \mathcal{I}_{5}} \lambda_{i}^{G} \nabla G_{i}\left(x^{*}\right)-\sum_{i \in \mathcal{I}_{3} \cup \mathcal{I}_{6}} \lambda_{i}^{H} \nabla H_{i}\left(x^{*}\right)+\eta^{*} .
$$

By RCPLD(ii), since the vectors $\lambda_{\mathcal{I}_{4}}^{g}, \lambda_{\mathcal{I}_{1}}^{h}, \lambda_{\mathcal{I}_{2} \cup \mathcal{I}_{5}}^{G}, \lambda_{\mathcal{I}_{3} \cup \mathcal{I}_{6}}^{H}, \eta^{*}$ are not all equal to zero, $x^{k} \rightarrow$ $x^{*}, v_{i}^{k} \rightarrow v_{i}^{*}, \frac{\alpha_{i}^{k} \eta_{i}^{k}}{M_{k}} \rightarrow \eta_{i}^{*}, i \in L$, the set of vectors

$$
\left\{v_{i}^{k}\right\}_{i \in \mathcal{I}_{4}} \cup\left\{\nabla h_{i}\left(x^{k}\right)\right\}_{i \in \mathcal{I}_{1}} \cup\left\{\nabla G_{i}\left(x^{k}\right)\right\}_{i \in \mathcal{I}_{2} \cup \mathcal{I}_{5}} \cup\left\{\nabla H_{i}\left(x^{k}\right)\right\}_{i \in \mathcal{I}_{3} \cup \mathcal{I}_{6}} \cup\left\{\frac{\alpha_{i}^{k} \nu_{i}^{k}}{M_{k}}\right\}_{i \in L}
$$

must be linearly dependent. Since $\alpha_{i}^{k} / M_{k}>0$ for each $i \in L$, this is a contradiction to the conclusion in step 2. The contradiction proves that $\left\{\left(\bar{\lambda}_{k}^{g}, \bar{\lambda}_{k}^{h}, \bar{\lambda}_{k}^{G}, \bar{\lambda}_{k}^{H}, \sum_{i \in L} \alpha_{i}^{k} \nu_{i}^{k}\right)\right\}$ is bounded.

Without loss of generality, assume that $\left(\bar{\lambda}_{k}^{g}, \bar{\lambda}_{k}^{h}, \bar{\lambda}_{k}^{G}, \bar{\lambda}_{k}^{H}, \sum_{i \in L} \alpha_{i}^{k} \nu_{i}^{k}\right) \rightarrow\left(\lambda^{g}, \lambda^{h}, \lambda^{G}, \lambda^{H}, \sum_{i \in L} \nu_{i}^{*}\right)$ as $k \rightarrow \infty$. It is easy to see that either $\lambda_{i}^{G}>0, \lambda_{i}^{H}>0$ or $\lambda_{i}^{G} \lambda_{i}^{H}=0 \quad \forall i \in \mathcal{J}^{*}, \lim _{k \rightarrow \infty} \sum_{i \in L} \alpha_{i}^{k} \nu_{i}^{k}=$ $\sum_{i \in L} \nu_{i}^{*} \in \mathcal{N}_{C}\left(x^{*}\right)$. Taking limits for a subsequence in (3.5), we derive that $x^{*}$ is an M-stationary point of $(\mathrm{P})$.

We now perform the next task of proving that RCPLD is a sufficient condition for the error bound property under extra regularity conditions. First we prove the following result which shows that RCPLD is persistent locally. 
Proposition 3.1 Assume that RCPLD holds at a feasible point $x^{*}$ of the system

$$
g(x) \leq 0, \quad h(x)=0, \quad x \in C .
$$

Then RCPLD holds at every point belongs to a sufficiently small neighborhood of $x^{*}$.

Proof. Consider any sequence $x^{k} \rightarrow x^{*}$ as $k \rightarrow \infty$. It is obvious that RCPLD (i) holds at each $x^{k}$ when $k$ is sufficiently large. Assume that $\left\{\nabla h_{i}\left(x^{*}\right)\right\}_{i \in \mathcal{I}_{1}}$ with $\mathcal{I}_{1} \subseteq\{1, \cdots, m\}$ is a basis for $\operatorname{span}\left\{\nabla h_{i}\left(x^{*}\right)\right\}_{i=1}^{m}$. Then $\left\{\nabla h_{i}\left(x^{k}\right)\right\}_{i \in \mathcal{I}_{1}}$ is a basis of $\operatorname{span}\left\{\nabla h_{i}\left(x^{k}\right)\right\}_{i=1}^{m}$ for any large $k$. We now show that RCPLD (ii) holds at $x^{k}$ for any sufficiently large $k$. To contrary, assume that RCPLD(ii) does not hold at each point of a subsequence $\left\{x^{k}\right\}_{k \in K_{0}}$. Then there exist an index set $\mathcal{I}_{2}^{k} \subseteq I_{g}^{k}:=\left\{i=1, \cdots, n: g_{i}\left(x^{k}\right)=0\right\}, v_{i}^{k} \in \partial g_{i}\left(x^{k}\right), i \in \mathcal{I}_{2}^{k}$ and a nonzero vector $\left(\lambda_{k}^{g}, \lambda_{k}^{h}, \eta^{k}\right)$ with $\lambda_{\mathcal{I}_{2}^{k}, k}^{g} \geq 0, \eta^{k}:=\left(\eta_{1}^{k}, \ldots, \eta_{l}^{k}\right) \in \mathcal{N}_{C}\left(x^{k}\right)$ such that

$$
0=\sum_{i \in \mathcal{I}_{2}^{k}} \lambda_{i, k}^{g} v_{i}^{k}+\sum_{i \in \mathcal{I}_{1}} \lambda_{i, k}^{h} \nabla h_{i}\left(x^{k}\right)+\eta^{k}
$$

but the set of vectors $\left\{v_{i}^{k, s}\right\}_{i \in \mathcal{I}_{2}^{k}} \cup\left\{\nabla h_{i}\left(y^{k, s}\right)\right\}_{i \in \mathcal{I}_{1}} \cup\left\{\nu_{i}^{k, s}\right\}_{i \in L}$, where $L:=\left\{1, \ldots, l: \eta_{i}^{k, s} \neq 0\right\}$, is linearly independent for some sequences $y^{k, s} \rightarrow x^{k}$ with $\partial g_{i}\left(y^{k, s}\right) \ni v_{i}^{k, s} \rightarrow v_{i}^{k}\left(i \in \mathcal{I}_{2}^{k}\right)$, $\nu_{i}^{k, s}:=\{0\}^{s_{i}} \times\left\{\eta_{i}^{k, s}\right\} \times\{0\}^{t_{i}}, \eta_{i}^{k, s} \in \mathcal{N}_{C_{i}}\left(y_{i}^{k, s}\right), \eta_{i}^{k, s} \rightarrow \eta_{i}^{k}$ as $s \rightarrow \infty$. Since $\mathcal{I}_{2}^{k} \subseteq I_{g}^{k} \subseteq I_{g}^{*}$ is a finite set, we may consider a subsequence such that $\mathcal{I}_{2}^{k}=\mathcal{I}_{2}$ for every large $k \in K_{0}$. Let $M_{k}:=\left\|\lambda_{k}^{g}\right\|+\left\|\lambda_{k}^{h}\right\|+\left\|\eta^{k}\right\|$. Suppose there exists a subsequence $K \subseteq K_{0}$ such that

$$
\lim _{k \rightarrow \infty, k \in K} \frac{\left(\lambda_{k}^{g}, \lambda_{k}^{h}, \eta^{k}\right)}{M_{k}}=\left(\lambda^{g}, \lambda^{h}, \eta\right)
$$

with $\lambda_{i}^{g} \geq 0, i \in \mathcal{I}_{2}$. Without loss of generality, assume that $v_{i}^{k} \rightarrow v_{i} \in \partial g_{i}\left(x^{*}\right)$ and we assume $\lim _{k \rightarrow \infty, k \in K} \frac{\eta_{i}^{k}}{M_{k}}=\eta_{i} \in \mathcal{N}_{C_{i}}\left(x_{i}^{*}\right), i=1, \cdots, l$. Since $\eta_{i}^{k, s} \rightarrow \eta_{i}^{k}$ as $s \rightarrow \infty$, we also have $\frac{\eta_{i}^{k, s}}{M_{k}} \rightarrow \eta_{i}, i \in L$ as $k, s \rightarrow \infty$.

By the diagonalization law, there exists a sequence $\left\{z^{k}\right\}$ converging to $x^{*}$ such that for each $k, \bar{v}_{i}^{k} \in \partial g_{i}\left(z^{k}\right), \bar{v}_{i}^{k} \rightarrow v_{i}^{k}, i=1, \cdots, n, \bar{\eta}_{i}^{k} \in \mathcal{N}_{C_{i}}\left(z_{i}^{k}\right), \frac{\bar{\eta}_{i}^{k}}{M_{k}} \rightarrow \eta_{i}, i \in L$ and $\left\{\bar{v}_{i}^{k}\right\}_{i \in \mathcal{I}_{2}} \cup$ $\left\{\nabla h_{i}\left(z^{k}\right)\right\}_{i \in \mathcal{I}_{1}} \cup\left\{\bar{\nu}_{i}^{k}\right\}_{i \in L}$ is linearly independent for all large $k$ and $\bar{\nu}_{i}^{k}:=\{0\}^{s_{i}} \times\left\{\bar{\eta}_{i}^{k}\right\} \times\{0\}^{t_{i}}$.

Dividing by $M_{k}$ in both sides of (3.7) and letting $k \rightarrow \infty, k \in K$, we have that $\eta \in \mathcal{N}_{C}\left(x^{*}\right)$ and

$$
0=\sum_{i \in \mathcal{I}_{2}} \lambda_{i}^{g} v_{i}+\sum_{i \in \mathcal{I}_{1}} \lambda_{i}^{h} \nabla h_{i}\left(x^{*}\right)+\eta
$$

From RCPLD (ii), the set of vectors $\left\{\bar{v}_{i}^{k}\right\}_{i \in \mathcal{I}_{2}} \cup\left\{\nabla h_{i}\left(z^{k}\right)\right\}_{i \in \mathcal{I}_{1}} \cup\left\{\frac{\bar{\nu}_{i}^{k}}{M_{k}}\right\}_{i \in L}$ must be linearly dependent, which is a contradiction. The contradiction shows that RCPLD holds at $x^{k}$ for $k$ sufficiently large. 
Theorem 3.2 Assume the RCPLD holds at a feasible point $x^{*}$ of the system (3.6), $g_{i}(\cdot), i=$ $1, \cdots, n$ are subdifferentially regular and $C$ is Clarke regular around $x^{*}$, then there exist $\alpha>0$ and $\varepsilon>0$ such that

$$
d_{\mathcal{F}_{1}}(x) \leq \alpha\left(\left\|g_{+}(x)\right\|+\|h(x)\|\right), \quad \forall x \in \mathbb{B}_{\varepsilon}\left(x^{*}\right) \cap C,
$$

where $\mathcal{F}_{1}$ denotes the set of feasible points satisfying system (3.6).

Proof. If $x^{*}$ is an interior point of $\mathcal{F}_{1}$, then $d_{\mathcal{F}_{1}}(x)=0$ for all $x \in \mathbb{B}_{\varepsilon}\left(x^{*}\right) \cap C$ and hence the result holds automatically. Now assume that $x^{*}$ lies in the boundary of $\mathcal{F}_{1}$. Let $\phi_{1}(x):=$ $\left\|g_{+}(x)\right\|+\|h(x)\|$. We rewrite the feasible set by $\mathcal{F}_{1}:=\left\{x \in C: \phi_{1}(x)=0\right\}$. Assume for a contradiction that there exists $C \ni x^{k} \rightarrow x^{*}$ such that $d_{\mathcal{F}_{1}}\left(x^{k}\right)>k \phi_{1}\left(x^{k}\right)$.

Obviously $x^{k} \notin \mathcal{F}_{1}$. Let $y^{k}$ be the projector of $x^{k}$ to $\mathcal{F}_{1}$. Then $d_{\mathcal{F}_{1}}\left(x^{k}\right)=\left\|y^{k}-x^{k}\right\| \neq 0$ and $\lim _{k \rightarrow \infty} y^{k}=x^{*}$. For each $k, y^{k}$ is an optimal solution of the following problem:

$$
\begin{array}{rll}
\left(\mathrm{P}_{k}^{\prime}\right) \quad \min & F^{k}(x):=\left\|x-x^{k}\right\| \\
\text { s.t. } & g(x) \leq 0, h(x)=0, x \in C,
\end{array}
$$

where $\|\cdot\|$ denotes the 2-norm. Since the RCPLD persists in a neighborhood of $x^{*}$, RCPLD holds at $y^{k}$ for $k$ sufficiently large. From Theorem 3.1 $y^{k}$ is a limiting stationary point of $\left(\mathrm{P}_{k}^{\prime}\right)$. By the optimality condition, there exist parameters $\lambda_{i, k}^{g} \geq 0, v_{i}^{k} \in \partial g_{i}\left(y^{k}\right)$ for $i \in I\left(y^{k}\right)$ and $\lambda_{i, k}^{h}, i=1, \cdots, m$ such that

$$
0=\frac{y^{k}-x^{k}}{\left\|y^{k}-x^{k}\right\|}+\sum_{i \in I\left(y^{k}\right)} \lambda_{i, k}^{g} v_{i}^{k}+\sum_{i=1}^{m} \lambda_{i, k}^{h} \nabla h_{i}\left(y^{k}\right)+\eta^{k} .
$$

Let $\nu_{i}^{k}:=\{0\}^{s_{i}} \times\left\{\eta_{i}^{k}\right\} \times\{0\}^{t_{i}}, \sum_{i \in L_{k}} \nu_{i}^{k}=\eta^{k} \in \mathcal{N}_{C}\left(y^{k}\right)$ and $L_{k}:=\left\{1, \cdots, l: \eta_{i}^{k} \neq 0\right\}$.

Assume that $\left\{\nabla h_{i}\left(x^{*}\right)\right\}_{i \in \mathcal{I}_{1}}$ with $\mathcal{I}_{1} \subseteq\{1, \cdots, m\}$ is a basis for $\operatorname{span}\left\{\nabla h_{i}\left(x^{*}\right)\right\}_{i=1}^{m}$. From Lemma 2.2, we obtain $\mathcal{I}_{2}^{k} \subseteq I\left(y^{k}\right) \cap \operatorname{supp}\left(\lambda_{i, k}^{g}\right)$ and $L_{k}^{\prime} \subseteq L_{k}$ with $\left\{\bar{\lambda}_{k}^{g}, \bar{\lambda}_{k}^{h}, \alpha^{k}\right\}, \bar{\lambda}_{i, k}^{g}>0$ for $i \in \mathcal{I}_{2}^{k}$ and $\alpha_{i}^{k}>0, i \in L_{k}^{\prime}$ such that

$$
0=\frac{y^{k}-x^{k}}{\left\|y^{k}-x^{k}\right\|}+\sum_{i \in \mathcal{I}_{2}^{k}} \bar{\lambda}_{i, k}^{g} v_{i}^{k}+\sum_{i \in \mathcal{I}_{1}} \bar{\lambda}_{i, k}^{h} \nabla h_{i}\left(y^{k}\right)+\sum_{i \in L_{k}^{\prime}} \alpha_{i}^{k} \nu_{i}^{k}
$$

with bounded multipliers $\left\{\left(\bar{\lambda}_{k}^{g}, \bar{\lambda}_{k}^{h}, \xi^{k}\right)\right\}$ from Theorem [3.1, for $k \rightarrow \infty, \xi^{k}:=\sum_{i \in L_{k}^{\prime}} \alpha_{i}^{k} \nu_{i}^{k} \in$ $\mathcal{N}_{C}\left(y^{k}\right)$. Let $M \geq\left\|\left(\bar{\lambda}_{k}^{g}, \bar{\lambda}_{k}^{h}\right)\right\|_{1}$ for sufficiently $k$.

From the subdifferentially regularity of $g_{i}(\cdot), i=1, \cdots, n$, for sufficiently large $k$,

$$
g_{i}\left(x^{k}\right)-g_{i}\left(y^{k}\right)-\left\langle v_{i}^{k}, x^{k}-y^{k}\right\rangle+\frac{1}{4 M}\left\|x^{k}-y^{k}\right\| \geq 0 .
$$

Similarly, $\left\langle\xi^{k}, x^{k}-y^{k}\right\rangle \leq \frac{1}{4}\left\|y^{k}-x^{k}\right\|$. Furthermore, for each $i=1, \cdots, m$,

$$
h_{i}\left(x^{k}\right)=h_{i}\left(y^{k}\right)+\left\langle\nabla h_{i}\left(y^{k}\right), x^{k}-y^{k}\right\rangle+o\left(\left\|x^{k}-y^{k}\right\|\right) .
$$


Then from (3.9), we have

$$
\begin{aligned}
\left\|y^{k}-x^{k}\right\|= & \left\langle\sum_{i \in \mathcal{I}_{2}^{k}} \bar{\lambda}_{i, k}^{g} v_{i}^{k}+\sum_{i \in \mathcal{I}_{1}} \bar{\lambda}_{i, k}^{h} \nabla h_{i}\left(y^{k}\right)+\xi^{k}, x^{k}-y^{k}\right\rangle \\
\leq & \sum_{i \in \mathcal{I}_{2}^{k}} \bar{\lambda}_{i, k}^{g}\left(g_{i}\left(x^{k}\right)-g_{i}\left(y^{k}\right)\right)+\sum_{i \in \mathcal{I}_{1}} \bar{\lambda}_{i, k}^{h}\left(h_{i}\left(x^{k}\right)-h_{i}\left(y^{k}\right)\right) \\
& +\frac{1}{4}\left\|y^{k}-x^{k}\right\|+\left(\sum_{i \in \mathcal{I}_{2}^{k}} \bar{\lambda}_{i, k}^{g}+\sum_{i \in \mathcal{I}_{1}}\left|\bar{\lambda}_{i, k}^{h}\right|\right) \frac{1}{4 M}\left\|y^{k}-x^{k}\right\| \\
\leq & \sum_{i \in \mathcal{I}_{2}^{k}} \bar{\lambda}_{i, k}^{g} g_{i}\left(x^{k}\right)+\sum_{i \in \mathcal{I}_{1}} \bar{\lambda}_{i, k}^{h} h_{i}\left(x^{k}\right)+\frac{1}{2}\left\|y^{k}-x^{k}\right\| .
\end{aligned}
$$

This means

$$
\begin{aligned}
d_{\mathcal{F}_{1}}\left(x^{k}\right)=\left\|y^{k}-x^{k}\right\| & \leq 2 M\left(\sum_{i \in \mathcal{I}_{2}^{k}} \max \left\{0, g_{i}\left(x^{k}\right)\right\}+\sum_{i \in \mathcal{I}_{1}}\left|h_{i}\left(x^{k}\right)\right|\right) \\
& \leq 2 M\left(\left\|\max \left\{0, g\left(x^{k}\right)\right\}\right\|_{1}+\left\|h\left(x^{k}\right)\right\|_{1}\right),
\end{aligned}
$$

which is a contradiction. Therefore the error bound property holds.

The error bound property for the general system (1.1) can be now obtained from Theorem 3.2 . It extends [14, Theorem 5.1] to allow nonsmooth inequality constraints and abstract constraints.

Corollary 3.1 Assume the RCPLD holds at $x^{*} \in \mathcal{F}, g_{i}(\cdot), i=1, \cdots, n$ are subdifferentially regular and $C$ is Clarke regular around $x^{*}$, the constraint $(G(x), H(x)) \in \Omega^{p}$ satisfies the strict complementarity condition at $x^{*}$, then there exist $\alpha>0$ and $\varepsilon>0$ such that

$$
d_{\mathcal{F}}(x) \leq \alpha \phi(x), \quad \forall x \in \mathbb{B}_{\varepsilon}\left(x^{*}\right) \cap C,
$$

where $\phi(x):=\left\|g_{+}(x)\right\|+\|h(x)\|+\sum_{i \in \mathcal{I}^{*}}\left|G_{i}(x)\right|+\sum_{i \in \mathcal{K}^{*}}\left|H_{i}(x)\right|$ and $\mathcal{F}$ denotes the set of feasible points satisfying system (1.1).

Proof. Since the strict complementarity holds at $x^{*}$, we have $\mathcal{J}^{*}=\emptyset$. Hence for all $x \in \mathcal{F}$ sufficiently close to $x^{*}$, we can represent it as a solution to the system

$$
g_{i}(x) \leq 0, i=1, \cdots, n, h_{i}(x)=0, i=1, \cdots, m, G_{i}(x)=0, i \in \mathcal{I}^{*}, H_{i}(x)=0, i \in \mathcal{K}^{*}, x \in C .
$$

Then the error bound property follows by Theorem 3.2 and the equivalence of the finite dimensional norm.

\section{Sufficient conditions for RCPLD}

Note that although the RCPLD is a weak condition, it may not be easy to verify. In this section we investigate sufficient conditions for RCPLD which are stronger but easier to verify.

It is easy to see that the following well-known constraint qualification implies RCPLD. 
Definition 4.1 Let $x^{*} \in \mathcal{F}$. We say that the no nonzero abnormal multiplier constraint qualification (NNAMCQ) holds at $x^{*}$ if there is no nonzero vector $\left(\lambda^{g}, \lambda^{h}, \lambda^{G}, \lambda^{H}, \eta^{*}\right) \in \mathbb{R}^{n} \times \mathbb{R}^{m} \times$ $\mathbb{R}^{p} \times \mathbb{R}^{p} \times \mathbb{R}^{d}$ satisfying $\lambda_{i}^{g} \geq 0$ for $i \in I_{g}^{*}$, and either $\lambda_{i}^{G}>0, \lambda_{i}^{H}>0$ or $\lambda_{i}^{G} \lambda_{i}^{H}=0, \forall i \in \mathcal{J}^{*}$, $\eta^{*} \in \mathcal{N}_{C}\left(x^{*}\right), v_{i}^{*} \in \partial g_{i}\left(x^{*}\right)$ for $i \in I_{g}^{*}$ such that

$$
0=\sum_{i \in I_{g}^{*}} \lambda_{i}^{g} v_{i}^{*}+\sum_{i=1}^{m} \lambda_{i}^{h} \nabla h_{i}\left(x^{*}\right)-\sum_{i \in \mathcal{I}^{*} \cup \mathcal{J}^{*}} \lambda_{i}^{G} \nabla G_{i}\left(x^{*}\right)-\sum_{i \in \mathcal{K}^{*} \cup \mathcal{J}^{*}} \lambda_{i}^{H} \nabla H_{i}\left(x^{*}\right)+\eta^{*} .
$$

It is obvious that when the rank of $\left\{\nabla h_{i}\left(x^{*}\right)\right\}_{i=1}^{m} \cup\left\{\nabla G_{i}\left(x^{*}\right)\right\}_{i \in \mathcal{I}^{*}} \cup\left\{\nabla H_{i}\left(x^{*}\right)\right\}_{i \in \mathcal{K}^{*}}$ is equal to $d$, RCPLD holds automatically. This condition is easy to verify and moreover it is not just a constraint qualification but also a sufficient condition for error bounds without imposing any regularity conditions.

Theorem 4.1 For a feasible point $x^{*} \in \mathcal{F}$, suppose that the rank of $\left\{\left\{\nabla h_{i}\left(x^{*}\right)\right\}_{i=1}^{m} \cup\left\{\nabla G_{i}\left(x^{*}\right)\right\}_{i \in \mathcal{I}^{*}} \cup\right.$ $\left.\left\{\nabla H_{i}\left(x^{*}\right)\right\}_{i \in \mathcal{K}^{*}}\right\}$ is equal to $d$. Then the error bound property (1.3) holds at $x^{*}$.

Proof. Around the point $x^{*}$, we can equivalently formulate the complementarity system $(G(x), H(x)) \in \Omega^{p}$ as

$$
G_{i}(x)=0, i \in \mathcal{I}^{*}, H_{i}(x)=0, i \in \mathcal{K}^{*},\left(G_{i}(x), H_{i}(x)\right) \in \Omega, i \in \mathcal{J}^{*} .
$$

Hence the constraints $G_{i}(x)=0, i \in \mathcal{I}^{*}, H_{i}(x)=0, i \in \mathcal{K}^{*}$ can be treated as equality constraints. We denote by $\phi(x):=\max \left\{0, g_{i}(x), i=1, \cdots, n,\left|h_{i}(x)\right|, i=1, \cdots, m,\left|G_{i}(x)\right|, i \in \mathcal{I}^{*},\left|H_{i}(x)\right|, i \in\right.$ $\left.\mathcal{K}^{*}, d_{\Omega}\left(G_{i}(x), H_{i}(x)\right), i \in \mathcal{J}^{*}\right\}$.

If $x^{*}$ is an interior point of $\mathcal{F}$, then $d_{\mathcal{F}}(x)=0$ for all $x \in \mathbb{B}_{\varepsilon}\left(x^{*}\right) \cap C$ and hence the result holds automatically. Now assume that $x^{*}$ lies in the boundary of $\mathcal{F}$. We rewrite the feasible set by $\mathcal{F}:=\{x \in C: \phi(x)=0\}$. To a contrary, assume that there exists $C \ni x^{k} \rightarrow x^{*}$ such that

$$
d_{\mathcal{F}}\left(x^{k}\right)>k \phi\left(x^{k}\right)
$$

For any $i=1, \cdots, m, h_{i}\left(x^{*}\right)=0$ and by the Taylor expansion,

$$
h_{i}\left(x^{k}\right)=h_{i}\left(x^{*}\right)+\left\langle\nabla h_{i}\left(x^{*}\right), x^{k}-x^{*}\right\rangle+o\left(\left\|x^{k}-x^{*}\right\|\right) .
$$

From (4.1), $\left\|x^{*}-x^{k}\right\| \geq d_{\mathcal{F}}\left(x^{k}\right)>k \phi\left(x^{k}\right) \geq k\left|h_{i}\left(x^{k}\right)\right|$, which implies that $\lim _{k \rightarrow \infty} \frac{h_{i}\left(x^{k}\right)}{\left\|x^{*}-x^{k}\right\|}=0$. Taking subsequence if necessary, let $d^{*}:=\lim _{k \rightarrow \infty} \frac{x^{k}-x^{*}}{\left\|x^{k}-x^{*}\right\|}$. Dividing the both sides of (4.2) by $\left\|x^{k}-x^{*}\right\|$ and letting $k \rightarrow \infty$, we have $\left\langle\nabla h_{i}\left(x^{*}\right), d^{*}\right\rangle=0$. Similarly from the above discussion, we have $\left\langle\nabla G_{i}\left(x^{*}\right), d^{*}\right\rangle=0, \forall i \in \mathcal{I}^{*}, \quad\left\langle\nabla H_{i}\left(x^{*}\right), d^{*}\right\rangle=0, \forall i \in \mathcal{K}^{*}$. This means that $d^{*}$ is linearly independent with all vectors in $\operatorname{span}\left\{\left\{\nabla h_{i}\left(x^{*}\right)\right\}_{i=1}^{m} \cup\left\{\nabla G_{i}\left(x^{*}\right)\right\}_{i \in \mathcal{I}^{*}} \cup\left\{\nabla H_{i}\left(x^{*}\right)\right\}_{i \in \mathcal{K}^{*}}\right\}$. Since by assumption the rank of span $\left\{\left\{\nabla h_{i}\left(x^{*}\right)\right\}_{i=1}^{m} \cup\left\{\nabla G_{i}\left(x^{*}\right)\right\}_{i \in \mathcal{I}^{*}} \cup\left\{\nabla H_{i}\left(x^{*}\right)\right\}_{i \in \mathcal{K}^{*}}\right\}$ is $d$, this is impossible since these vectors lie in $\mathbb{R}^{d}$. The proof of the theorem is therefore complete. 
In the following definition we extend the well-known concept of RCRCQ (see [19] for the smooth equality and inequality systems and [11, Definition 3.4] for system with complementarity constraints) to our general system (1.1). RCRCQ is stronger than the RCPLD but may be easier to verify.

Definition 4.2 Let $x^{*} \in \mathcal{F}$. We say that the relaxed constant rank constraint qualification (RCRCQ) holds at $x^{*}$ if for all sufficiently large $k$, any index sets $\mathcal{I}_{4} \subseteq I_{g}^{*}, \mathcal{I}_{5} \subseteq \mathcal{J}^{*}, \mathcal{I}_{6} \subseteq \mathcal{J}^{*}$, $\mathcal{L} \subseteq\{1, \cdots, l\}$ and any vectors $v_{i}^{*} \in \partial g_{i}\left(x^{*}\right)\left(i \in \mathcal{I}_{4}\right), \eta_{i}^{*} \in \mathcal{N}_{C_{i}}\left(x_{i}^{*}\right)(i \in \mathcal{L}), \nu_{i}^{*}:=\{0\}^{s_{i}} \times\left\{\eta_{i}^{*}\right\} \times$ $\{0\}^{t_{i}}$ with $s_{i}:=q_{1}+\cdots+q_{i-1}$ and $t_{i}:=q_{i+1}+\cdots+q_{l}$, the set of vectors

$$
\left\{v_{i}^{*}\right\}_{i \in \mathcal{I}_{4}} \cup\left\{\nabla h_{i}\left(x^{*}\right)\right\}_{i=1}^{m} \cup\left\{\nabla G_{i}\left(x^{*}\right)\right\}_{i \in \mathcal{I}^{*} \cup \mathcal{I}_{5}} \cup\left\{\nabla H_{i}\left(x^{*}\right)\right\}_{i \in \mathcal{K}^{*} \cup \mathcal{I}_{6}} \cup\left\{\nu_{i}^{*}\right\}_{i \in \mathcal{L}},
$$

and the set of vectors

$$
\left\{v_{i}^{k}\right\}_{i \in \mathcal{I}_{4}} \cup\left\{\nabla h_{i}\left(x^{k}\right)\right\}_{i=1}^{m} \cup\left\{\nabla G_{i}\left(x^{k}\right)\right\}_{i \in \mathcal{I}^{*} \cup \mathcal{I}_{5}} \cup\left\{\nabla H_{i}\left(x^{k}\right)\right\}_{i \in \mathcal{K}^{*} \cup \mathcal{I}_{6}} \cup\left\{\nu_{i}^{k}\right\}_{i \in \mathcal{L}},
$$

where $x^{k} \neq x^{*}, \nu_{i}^{k}:=\{0\}^{s_{i}} \times\left\{\eta_{i}^{k}\right\} \times\{0\}^{t_{i}}$, have the same rank for all sequences $\left\{x^{k}\right\},\left\{v^{k}\right\},\left\{\eta^{k}\right\}$ satisfying $x^{k} \rightarrow x^{*}, v^{k} \rightarrow v^{*}, \eta^{k} \rightarrow \eta^{*}$ as $k \rightarrow \infty, v_{i}^{k} \in \partial g_{i}\left(x^{k}\right), \eta_{i}^{k} \in \mathcal{N}_{C_{i}}\left(x_{i}^{k}\right)$.

Proposition 4.1 RCRCQ implies RCPLD.

Proof. Let $x^{*} \in \mathcal{F}$ satisfy RCRCQ. Taking $\mathcal{I}_{4} \cup \mathcal{I}_{5} \cup \mathcal{I}_{6} \cup \mathcal{L}=\emptyset$, we have that RCPLD (i) holds. We now show the RCPLD (ii) holds at $x^{*}$. Let $\mathcal{I}_{1} \subseteq\{1, \cdots, m\}, \mathcal{I}_{2} \subseteq \mathcal{I}^{*}, \mathcal{I}_{3} \subseteq \mathcal{K}^{*}$ be such that the set of vectors $\left\{\nabla h_{i}\left(x^{*}\right)\right\}_{i \in \mathcal{I}_{1}} \cup\left\{\nabla G_{i}\left(x^{*}\right)\right\}_{i \in \mathcal{I}_{2}} \cup\left\{\nabla H_{i}\left(x^{*}\right)\right\}_{i \in \mathcal{I}_{3}}$ is a basis for $\operatorname{span}\left\{\left\{\nabla h_{i}\left(x^{*}\right)\right\}_{i=1}^{m} \cup\left\{\nabla G_{i}\left(x^{*}\right)\right\}_{i \in \mathcal{I}^{*}} \cup\left\{\nabla H_{i}\left(x^{*}\right)\right\}_{i \in \mathcal{K}^{*}}\right\}$.

Let $\mathcal{I}_{4} \subseteq I_{g}^{*}, \mathcal{I}_{5}, \mathcal{I}_{6} \subseteq \mathcal{J}^{*}$ be the index sets such that there exists a nonzero vector $\left(\lambda^{g}, \lambda^{h}, \lambda^{G}, \lambda^{H}, \eta^{*}\right)$ satisfying $\lambda_{i}^{g} \geq 0$ for $i \in \mathcal{I}_{4}$, and either $\lambda_{i}^{G}>0, \lambda_{i}^{H}>0$ or $\lambda_{i}^{G} \lambda_{i}^{H}=0, \forall i \in \mathcal{J}^{*}, \eta^{*}=\left(\eta_{1}^{*}, \cdots, \eta_{l}^{*}\right) \in$ $\mathcal{N}_{C}\left(x^{*}\right), v_{i}^{*} \in \partial g_{i}\left(x^{*}\right)$ for $i \in \mathcal{I}_{4}$ satisfying

$$
0=\sum_{i \in \mathcal{I}_{4}} \lambda_{i}^{g} v_{i}^{*}+\sum_{i \in \mathcal{I}_{1}} \lambda_{i}^{h} \nabla h_{i}\left(x^{*}\right)-\sum_{i \in \mathcal{I}_{2} \cup \mathcal{I}_{5}} \lambda_{i}^{G} \nabla G_{i}\left(x^{*}\right)-\sum_{i \in \mathcal{I}_{3} \cup \mathcal{I}_{6}} \lambda_{i}^{H} \nabla H_{i}\left(x^{*}\right)+\eta^{*} .
$$

Then the vectors

$$
\left\{v_{i}^{*}\right\}_{i \in \mathcal{I}_{4}} \cup\left\{\nabla h_{i}\left(x^{*}\right)\right\}_{i \in \mathcal{I}_{1}} \cup\left\{\nabla G_{i}\left(x^{*}\right)\right\}_{i \in \mathcal{I}_{2} \cup \mathcal{I}_{5}} \cup\left\{\nabla H_{i}\left(x^{*}\right)\right\}_{i \in \mathcal{I}_{3} \cup \mathcal{I}_{6}} \cup\left\{\nu_{i}^{*}\right\}_{i \in L},
$$

where $\nu_{i}^{*}:=\{0\}^{s_{i}} \times\left\{\eta_{i}^{*}\right\} \times\{0\}^{t_{i}}$ and $L:=\left\{1, \ldots, l: \eta_{i}^{*} \neq 0\right\}$, is linearly dependent. Since $\left\{\nabla h_{i}\left(x^{*}\right)\right\}_{i \in \mathcal{I}_{1}} \cup\left\{\nabla G_{i}\left(x^{*}\right)\right\}_{i \in \mathcal{I}_{2}} \cup\left\{\nabla H_{i}\left(x^{*}\right)\right\}_{i \in \mathcal{I}_{3}}$ is a basis for span $\left\{\left\{\nabla h_{i}\left(x^{*}\right)\right\}_{i=1}^{m} \cup\left\{\nabla G_{i}\left(x^{*}\right)\right\}_{i \in \mathcal{I}^{*}} \cup\right.$ $\left.\left\{\nabla H_{i}\left(x^{*}\right)\right\}_{i \in \mathcal{K}^{*}}\right\}$, it follows that

$$
\left\{v_{i}^{*}\right\}_{i \in \mathcal{I}_{4}} \cup\left\{\nabla h_{i}\left(x^{*}\right)\right\}_{i=1}^{m} \cup\left\{\nabla G_{i}\left(x^{*}\right)\right\}_{i \in \mathcal{I}^{*} \cup \mathcal{I}_{5}} \cup\left\{\nabla H_{i}\left(x^{*}\right)\right\}_{i \in \mathcal{K}^{*} \cup \mathcal{I}_{6}} \cup\left\{\nu_{i}^{*}\right\}_{i \in L}
$$

is linearly dependent as well. By RCRCQ, it follows that

$$
\left\{v_{i}^{k}\right\}_{i \in \mathcal{I}_{4}} \cup\left\{\nabla h_{i}\left(x^{k}\right)\right\}_{i=1}^{m} \cup\left\{\nabla G_{i}\left(x^{k}\right)\right\}_{i \in \mathcal{I}^{*} \cup \mathcal{I}_{5}} \cup\left\{\nabla H_{i}\left(x^{k}\right)\right\}_{i \in \mathcal{K}^{*} \cup \mathcal{I}_{6}} \cup\left\{\nu_{i}^{k}\right\}_{i \in L}
$$


is linearly dependent for all sequences $\left\{x^{k}\right\},\left\{v^{k}\right\},\left\{\eta^{k}\right\}$ satisfying $x^{k} \rightarrow x^{*}, v^{k} \rightarrow v^{*}, \eta^{k} \rightarrow \eta^{*}$, $v_{i}^{k} \in \partial g_{i}\left(x^{k}\right), \eta_{i}^{k} \in \mathcal{N}_{C_{i}}\left(x_{i}^{k}\right), \nu_{i}^{k}:=\{0\}^{s_{i}} \times\left\{\eta_{i}^{k}\right\} \times\{0\}^{t_{i}}$. From RCPLD (i), $\left\{\nabla h_{i}\left(x^{k}\right)\right\}_{i \in \mathcal{I}_{1}} \cup$ $\left\{\nabla G_{i}\left(x^{k}\right)\right\}_{i \in \mathcal{I}_{2}} \cup\left\{\nabla H_{i}\left(x^{k}\right)\right\}_{i \in \mathcal{I}_{3}}$ is a basis of $\operatorname{span}\left\{\left\{\nabla h_{i}\left(x^{k}\right)\right\}_{i=1}^{m} \cup\left\{\nabla G_{i}\left(x^{k}\right)\right\}_{i \in \mathcal{I}^{*}} \cup\left\{\nabla H_{i}\left(x^{k}\right)\right\}_{i \in \mathcal{K}^{*}}\right\}$ and thus

$$
\left\{v_{i}^{k}\right\}_{i \in \mathcal{I}_{4}} \cup\left\{\nabla h_{i}\left(x^{k}\right)\right\}_{i \in \mathcal{I}_{1}} \cup\left\{\nabla G_{i}\left(x^{k}\right)\right\}_{i \in \mathcal{I}_{2} \cup \mathcal{I}_{5}} \cup\left\{\nabla H_{i}\left(x^{k}\right)\right\}_{i \in \mathcal{I}_{3} \cup \mathcal{I}_{6}} \cup\left\{\nu_{i}^{k}\right\}_{i \in L}
$$

is linearly dependent. Since $L \subseteq L_{k}:=\left\{1, \ldots, l: \eta_{i}^{k} \neq 0\right\}$ for any sufficiently large $k$, RCPLD (ii) holds.

Definition 4.3 We say that the Linear Constraint Qualification (LCQ) holds if all functions $g_{i}, h_{i}, G_{i}, H_{i}$ are linear and the set $C$ is the union of finitely many polyhedral sets.

We now show that LCQ implies RCRCQ.

Proposition 4.2 LCQ implies RCRCQ holds at each $x^{*} \in \mathcal{F}$.

Proof. Since $C$ is the union of finitely many polyhedral convex sets, for all $x^{k} \rightarrow x^{*}$ and sufficiently large $k$, we have $\eta^{k}=\eta^{*}$ if $\eta^{k} \rightarrow \eta^{*}$ and $\eta^{k} \in \mathcal{N}_{C}\left(x^{k}\right)$. Moreover all functions are linear.

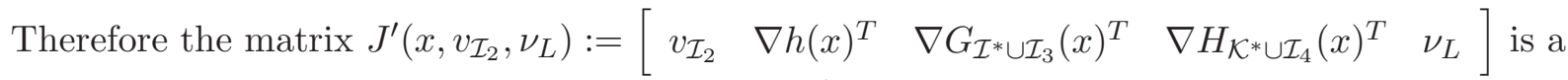
constant matrix for all $x, v_{\mathcal{I}_{2}}=\nabla g_{\mathcal{I}_{2}}(x), \eta \in\left\{\eta^{k}, \eta^{*}\right\}, \nu_{i}:=\{0\}^{s_{i}} \times\left\{\eta_{i}\right\} \times\{0\}^{t_{i}}, i \in L$ and therefore RCRCQ holds.

Example 4.1 Consider the nonsmooth system:

$$
\begin{aligned}
& h(x):=2 x_{1}+x_{2}=0, \\
& g(x):=x_{1}+x_{2}-\max \left\{\frac{1}{2}, x_{3}\right\}-x_{4}+1 \leq 0, \\
& x \in C:=C_{1} \times C_{2},
\end{aligned}
$$

where $C_{1}$ is the graph of a continuous function $\varphi:[-1,1] \rightarrow \mathbb{R}$ as shown in Fig.1 and $C_{2}:=$ $\{(t, 1-t): t \in[0,1]\}$. We set $\varphi(x)=0$ when $x=0,2^{-n},-2^{-n}$, for $n=0,1,2, \cdots$. Between any two points of the form $2^{-n-1}$ and $2^{-n}$, or $-2^{-n}$ and $-2^{-n-1}$, the graph of $\varphi$ describes the edge of an isosceles triangle whose apex is located at $\left(2^{-n-2}+2^{-n-1}, 1\right)$ or $\left(-2^{-n-2}-2^{-n-1}, 1\right)$. Consider the feasible solution $x^{*}=\left(x_{1}^{*}, x_{2}^{*}, x_{3}^{*}, x_{4}^{*}\right)=\left(0,0, \frac{1}{2}, \frac{1}{2}\right)$. We have $\nabla h(x)=(2,1,0,0)$,

$$
\partial g(x)= \begin{cases}(1,1,-1,-1) & \text { if } x_{3}>\frac{1}{2} \\ \{(1,1,-1,-1),(1,1,0,-1)\} & \text { if } x_{3}=\frac{1}{2} \\ (1,1,0,-1) & \text { if } x_{3}<\frac{1}{2}\end{cases}
$$

Since $C_{1}$ is symmetrical, we only give the expression of the normal cone for the case when $\left(x_{1}, x_{2}\right) \in C_{1}$ and $x_{1} \geq 0$, for $n=0,1,2, \cdots$,

$\mathcal{N}_{C_{1}}\left(x_{1}, x_{2}\right)=$ 


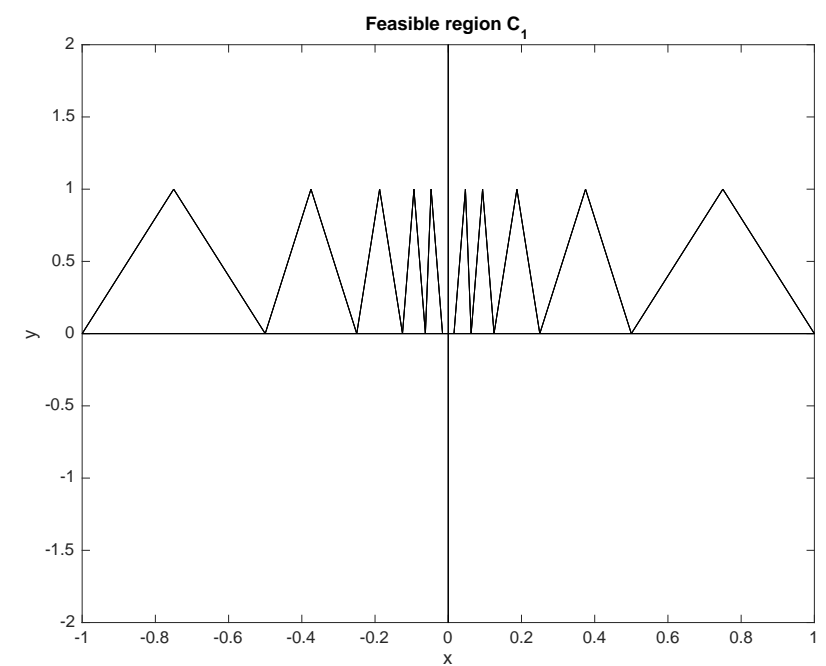

Figure 1: Feasible region $C_{1}$

$$
\left\{\begin{array}{lll}
\eta_{1}=0, \eta_{2} \in \mathbb{R} & \text { if } x_{1} \in\left(2^{-n-1}, 2^{-n}\right), x_{2}=0, \\
\eta_{2}=-2^{-n-2} \eta_{1}, \eta_{1} \in \mathbb{R} & \text { if } x_{1} \in\left(2^{-n-1}, 2^{-n-2}+2^{-n-1}\right), x_{2}>0, \\
& \eta_{2}=2^{-n-2} \eta_{1}, \eta_{1} \in \mathbb{R} & \text { if } x_{1} \in\left(2^{-n-2}+2^{-n-1}, 2^{-n}\right), x_{2}>0, \\
\left(\eta_{1}, \eta_{2}\right): & \eta_{2}=\alpha \eta_{1}, \alpha= \pm 2^{-n-2} \text { or } \eta_{2}>0 \text { with } & \\
\alpha \in\left(-\infty,-2^{-n-2}\right) \cup\left(2^{-n-2},+\infty\right) & \text { if } x_{1}=2^{-n-2}+2^{-n-1}, x_{2}=1, \\
\eta_{1}=0, \text { or } \eta_{2}=\alpha \eta_{1}, \alpha=-2^{-n-2}, 2^{-n-3} & \text { if } x_{1}=2^{-n-1}, x_{2}=0, \\
\eta_{1} \eta_{2}=0 & \text { if } x_{1}=0, x_{2}=0,
\end{array}\right.
$$

and

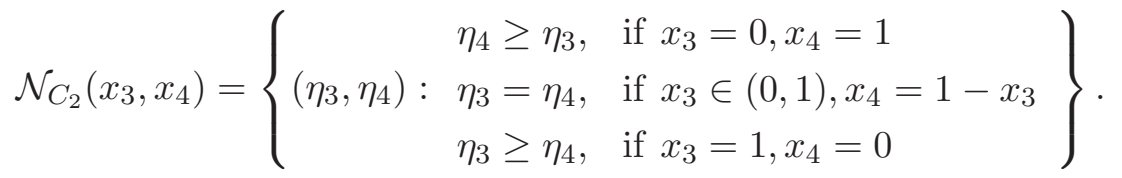

Also we have $\mathcal{N}_{C}(x)=\mathcal{N}_{C_{1}}\left(x_{1}, x_{2}\right) \times \mathcal{N}_{C_{2}}\left(x_{3}, x_{4}\right)$. Hence $\mathcal{N}_{C}\left(x^{*}\right)=\left\{\left(\eta_{1}, \eta_{2}\right): \eta_{1} \eta_{2}=0\right\} \times$ $\{(\alpha, \alpha): \alpha \in \mathbb{R}\}$. Let $\eta^{*}=(0,0,0,0)$ being an element in the normal cone $\mathcal{N}_{C}\left(x^{*}\right)$. For any $x^{k} \rightarrow x^{*}$ with $x_{1}^{k}=x_{2}^{k}=0, x_{3}^{k}>\frac{1}{2}, v^{k}=(1,1,-1,-1) \in \partial g\left(x^{k}\right), \eta^{k}=\left(0,0, \alpha_{k}, \alpha_{k}\right) \in \mathcal{N}_{C}\left(x^{k}\right)$ with $\alpha_{k} \rightarrow 0$, we have

$$
J^{\prime}\left(x^{k}, v^{k}, \eta^{k}\right):=\left[\begin{array}{lll}
v^{k} & \nabla h\left(x^{k}\right) & \eta^{k}
\end{array}\right]=\left[\begin{array}{ccc}
1 & 2 & 0 \\
1 & 1 & 0 \\
-1 & 0 & \alpha_{k} \\
-1 & 0 & \alpha_{k}
\end{array}\right] .
$$

$r\left(J^{\prime}\left(x^{k}, v^{k}, \eta^{k}\right)\right)=3$ if $\alpha_{k} \neq 0$ but $r\left(J^{\prime}\left(x^{*}, v^{*}, \eta^{*}\right)\right)=2$. This shows that RCRCQ fails at $x^{*}$.

Since the condition

$$
0=\lambda v^{*}+\mu \nabla h\left(x^{*}\right)+\eta^{*}
$$


holds when $\eta^{*}=(1,0,1,1) \in \mathcal{N}_{C}\left(x^{*}\right), v^{*}=(1,1,-1,-1) \in \partial g\left(x^{*}\right), \lambda=1=-\mu$. Hence the NNAMCQ fails at $x^{*}$.

We now verify that RCPLD holds. Actually it is easy to see that

$$
0=\lambda v^{*}+\mu \nabla h\left(x^{*}\right)+\eta^{*}, \quad v^{*} \in \partial g\left(x^{*}\right), \quad \eta^{*} \in \mathcal{N}_{C}\left(x^{*}\right)
$$

holds with $\mu, \lambda \geq 0, \eta^{*}$ not all equal to zero if and only if $v^{*}=(1,1,-1,-1)$ and either (a) or (b) below holds:

(a) $\lambda=-\mu, \eta^{*}=\mu(-1,0,-1,-1)$.

(b) $\lambda=-2 \mu, \eta^{*}=2 \mu\left(0,-\frac{1}{2}, 1,1\right)$.

In either case (a) or (b), the set $L=\{1,2\}$. For any $x^{k} \rightarrow x^{*}=\left(0,0, \frac{1}{2}, \frac{1}{2}\right)$, denote by $\eta^{k}:=$ $\left(\eta_{1}^{k}, \eta_{2}^{k}, \alpha_{k}, \alpha_{k}\right)$ where $\left(\eta_{1}^{k}, \eta_{2}^{k}\right) \in \mathcal{N}_{C_{1}}\left(x_{1}^{k}, x_{2}^{k}\right),\left(\alpha_{k}, \alpha_{k}\right) \in \mathcal{N}_{C_{2}}\left(x_{3}^{k}, x_{4}^{k}\right)$ and $\eta^{k} \rightarrow \eta^{*}$. We also denote by $\nu_{1}^{k}:=\left(\eta_{1}^{k}, \eta_{2}^{k}, 0,0\right), \nu_{2}^{k}:=\left(0,0, \alpha_{k}, \alpha_{k}\right)$. Note that at any $x^{k}$ such that $x^{k} \rightarrow x^{*}, v^{k} \rightarrow$ $v^{*}, v^{k} \in \partial g\left(x^{k}\right)$ and $k$ is large enough, $g(x)$ is differentiable and $v^{k}=\nabla g\left(x^{k}\right)=(1,1,-1,-1)$.

Since the matrix

$$
J^{\prime}\left(x^{k}, v^{k}, \nu_{L}^{k}\right)=\left[\begin{array}{cccc}
1 & 2 & \eta_{1}^{k} & 0 \\
1 & 1 & \eta_{2}^{k} & 0 \\
-1 & 0 & 0 & \alpha_{k} \\
-1 & 0 & 0 & \alpha_{k}
\end{array}\right]
$$

is not full rank, the set of vectors $\nabla h\left(x^{k}\right), v^{k}, \nu_{1}^{k}, \nu_{2}^{k}$ is always linearly dependent and thus RCPLD holds at $x^{*}$.

Note that if $\alpha_{k}-\eta_{1}^{k}+2 \eta_{2}^{k} \neq 0$ for large $k$, it is easy to see that the rank of the matrix

$$
\left[\begin{array}{ccc}
1 & 2 & \eta_{1}^{k} \\
1 & 1 & \eta_{2}^{k} \\
-1 & 0 & \alpha_{k} \\
-1 & 0 & \alpha_{k}
\end{array}\right]
$$

equals to 3 and thus the set of vectors $\nabla h\left(x^{k}\right), v^{k}, \eta^{k}$ is linearly independent. Hence as pointed out in Remark 1.1, our definition for RCPLD is weaker than the condition that the set of vectors $\nabla h\left(x^{k}\right), v^{k}, \eta^{k}$ is linearly dependent.

\section{Applications to bilevel programs}

In this section we apply RCPLD to the combined program (CP). Throughout this section we assume that the value function $V(x)$ is Lipschitz continuous at the point of interest. For the case where $Y(x)=Y$ is independent of $x$, we can use Danskin's theorem and for the general case one can use Proposition 5.2 which is a special case of [4, Theorem 6.5.2]. Other weaker 
sufficient conditions for Lipschitz continuity of the value function as well as the estimates for its subdifferentials can be found for examples in [12].

Proposition 5.1 (Danskin's Theorem) ([5, page 99] or [6]) Let $Y \subseteq \mathbb{R}^{s}$ be a compact set and $f(x, y)$ be a function defined on $\mathbb{R}^{d} \times \mathbb{R}^{s}$ that is continuously differentiable at $x^{*}$. Then the value function $V(x):=\min \{f(x, y): y \in Y\}$ is Lipschitz continuous near $x^{*}$ and its Clarke subdifferential is $\partial^{c} V\left(x^{*}\right)=c o\left\{\nabla_{x} f\left(x^{*}, y\right): y \in S\left(x^{*}\right)\right\}$, where $\partial^{c} V\left(x^{*}\right)=\operatorname{co} \partial V\left(x^{*}\right)$ is the Clarke subdifferential of $V$ at $x^{*}$.

Proposition 5.2 Assume that the set-valued map $Y(x)$ is uniformly bounded around $x^{*}$, i.e., there exists a neighborhood $\mathbb{U}$ of $x^{*}$ such that the set $\bigcup_{x \in \mathbb{U}} Y(x)$ is bounded. Suppose that $M F C Q$ holds at $y$ for all $y \in S\left(x^{*}\right)$. Then the valued function $V(x)$ is Lipschitz continuous near $x^{*}$ and

$$
\partial^{c} V\left(x^{*}\right) \subseteq \operatorname{coW}\left(x^{*}\right)
$$

where

$$
\begin{aligned}
W\left(x^{*}\right) & :=\bigcup_{y \in S\left(x^{*}\right)}\left\{\nabla_{x} f\left(x^{*}, y\right)+u \nabla_{x} g\left(x^{*}, y\right)+v \nabla_{x} h\left(x^{*}, y\right):(u, v) \in M\left(x^{*}, y\right)\right\}, \\
M\left(x^{*}, y\right) & :=\left\{(u, v): \begin{array}{c}
0=\nabla_{y} f\left(x^{*}, y\right)+u \nabla_{y} g\left(x^{*}, y\right)+v \nabla_{y} h\left(x^{*}, y\right) \\
u \geq 0, \quad\langle g(x, y), u\rangle=0
\end{array}\right\},
\end{aligned}
$$

where $u \nabla_{y} g:=\sum_{i=1}^{m} u_{i} \nabla_{y} g_{i}$.

Note that if in addition to the assumptions of Proposition [5.2. $S$ is inner semicontinuous at $\left(x^{*}, y^{*}\right)$ for some $y^{*} \in S\left(x^{*}\right)$, then the union $\bigcup_{y \in S\left(x^{*}\right)}$ sign can be omitted in Proposition [5.2, see [21, Corollary 1.109]. In the case where the LICQ holds at each $y \in S\left(x^{*}\right)$, the set of multipliers $M\left(x^{*}, y\right)$ is a singleton and by [9, Corollary 5.4], the inclusion becomes an equality in the above proposition and $-V(x)$ is Clarke regular around $x^{*}$. In this case, LICQ for the lower level problem holds at every $y, y \in S(x)$, for all $x$ near $x^{*}$ due to the outer semi-continuity of the solution mapping $S(x)$. Thus we have

$$
\partial_{x}(f-V)(x, y)=\partial_{x}^{c}(f-V)(x, y)=\nabla_{x} f(x, y)-\partial^{c} V(x)=\nabla_{x} f(x, y)-c o W(x) .
$$

By Carathéodory's theorem, the convex set $\operatorname{coW}(x) \subseteq \mathbb{R}^{d}$ can be represented by not more than $d+1$ elements. It follows that for any $w \in \operatorname{co} W(x)$, there exist $\mu^{i} \geq 0, \sum_{i=1}^{d+1} \mu^{i}=1$, $M\left(x, y^{i}\right)=\left\{\left(u^{i}, v^{i}\right)\right\}, y^{i} \in S(x)$ such that $w=\sum_{i=1}^{d+1} \mu^{i}\left(\nabla_{x} f\left(x, y^{i}\right)+u^{i} \nabla_{x} g\left(x, y^{i}\right)+v^{i} \nabla_{x} h\left(x, y^{i}\right)\right)$.

Given a feasible vector $\left(x^{*}, y^{*}, u^{*}, v^{*}\right)$ of the problem $(\mathrm{CP})$, define the following index sets:

$$
\begin{aligned}
& I_{G}^{*}=I_{G}\left(x^{*}, y^{*}\right):=\left\{i: G_{i}\left(x^{*}, y^{*}\right)=0\right\}, \\
& \mathcal{I}^{*}=\mathcal{I}\left(x^{*}, y^{*}, u^{*}\right):=\left\{i: g_{i}\left(x^{*}, y^{*}\right)=0, u_{i}^{*}>0\right\}, \\
& \mathcal{J}^{*}=\mathcal{J}\left(x^{*}, y^{*}, u^{*}\right):=\left\{i: g_{i}\left(x^{*}, y^{*}\right)=0, u_{i}^{*}=0\right\} \\
& \mathcal{K}^{*}=\mathcal{K}\left(x^{*}, y^{*}, u^{*}\right):=\left\{i: g_{i}\left(x^{*}, y^{*}\right)<0, u_{i}^{*}=0\right\} .
\end{aligned}
$$


Theorem 3.1 can now be applied to the problem (CP) to obtain the M-stationary condition under RCPLD at any local optimal solution.

Theorem 5.1 Let $\left(x^{*}, y^{*}, u^{*}, v^{*}\right)$ be a local solution of $(\mathrm{CP})$ and suppose that the value function $V(x)$ is Lipschitz continuous at $x^{*}$. If the RCPLD holds at $\left(x^{*}, y^{*}, u^{*}, v^{*}\right)$, then $\left(x^{*}, y^{*}, u^{*}, v^{*}\right)$ is an M-stationary point of problem (CP).

In the rest of this section, we apply the sufficient conditions for RCPLD which are introduced in Section 4 to the bilevel programs. The following theorem follows immediately from Theorem 4.1. The condition is easy to verify since the nonsmooth constraint $f(x, y)-V(x) \leq 0$ is not needed in the verification.

Theorem 5.2 Let $\left(x^{*}, y^{*}, u^{*}, v^{*}\right)$ be a local solution of $(\mathrm{CP})$ and suppose that the value function $V(x)$ is Lipschitz continuous at $x^{*}$. If the rank of the matrix

$$
J^{*}=\left[\begin{array}{ccc}
\nabla\left(\nabla_{y} f+u \nabla_{y} g+v \nabla_{y} h\right)\left(x^{*}, y^{*}\right) & \nabla_{y} h\left(x^{*}, y^{*}\right)^{T} & \nabla_{y} g_{\mathcal{I}^{*} \cup \mathcal{J}^{*}}\left(x^{*}, y^{*}\right)^{T} \\
\nabla h\left(x^{*}, y^{*}\right) & 0 & 0 \\
\nabla H\left(x^{*}, y^{*}\right) & 0 & 0 \\
\nabla g_{\mathcal{I}^{*}}\left(x^{*}, y^{*}\right) & 0 & 0
\end{array}\right]
$$

is equal to $d+s+m+n-\left|\mathcal{K}^{*}\right|$, then $R C P L D$ holds and $\left(x^{*}, y^{*}, u^{*}, v^{*}\right)$ is an M-stationary point of problem (CP). Moreover $\left(x^{*}, y^{*}, u^{*}, v^{*}\right)$ is a local optimal solution of the penalized problem for some $\mu \geq 0$ :

$$
\left(\mathrm{CP}_{\mu}\right) \quad \min _{x, y, u, v} F(x, y)+\mu \phi_{C P}(x, y, u, v)
$$

where $\phi_{C P}(x, y, u, v):=(f(x, y)-V(x))_{+}+\|H(x, y)\|+\|h(x, y)\|+\left\|G_{+}(x, y)\right\|+\left\|\nabla_{y} L(x, y, u, v)\right\|+$ $\left.\sum_{i=1}^{m} d_{\Omega}\left(-g_{i}(x, y), u_{i}\right)\right)$.

Proof. Let $\Gamma^{*}:=\left[\begin{array}{c}\nabla_{y} g_{\mathcal{K}^{*}}\left(x^{*}, y^{*}\right)^{T} \\ 0\end{array}\right]$. It is easy to see that

$$
r\left(\left[\begin{array}{cc}
J^{*} & \Gamma^{*} \\
0 & I_{\left|\mathcal{K}^{*}\right|}
\end{array}\right]\right)=r\left(J^{*}\right)+\left|\mathcal{K}^{*}\right|=d+s+m+n,
$$

where $I_{\left|\mathcal{K}^{*}\right|}$ is the identity matrix of size $\left|\mathcal{K}^{*}\right|$. From Theorem 4.1, the error bound property holds for the problem (CP), i.e., there exist $\alpha>0$ and $\varepsilon>0$ such that

$$
d_{\mathcal{F}_{C P}}(x, y, u, v) \leq \alpha \phi_{C P}(x, y, u, v), \quad \forall(x, y, u, v) \in \mathbb{B}_{\varepsilon}\left(x^{*}, y^{*}, u^{*}, v^{*}\right),
$$

where $\mathcal{F}_{C P}$ denotes the feasible region of problem $(\mathrm{CP})$. It follows from Clarke's exact penalty principle [4, Proposition 2.4.3] that the problem $\left(\mathrm{CP}_{\mu}\right)$ is exact with $\mu \geq L_{F} \alpha$ with $L_{F}$ being the Lipschitz constant of the function $F$. 
Corollary 5.1 Let $\left(x^{*}, y^{*}, u^{*}, v^{*}\right)$ be a local solution of $(\mathrm{CP})$. Suppose that the value function $V(x)$ is Lipschitz continuous at $x^{*}$ and LICQ holds at $y^{*}$. Suppose the matrix

$$
S J^{*}=\left[\begin{array}{l}
\nabla h\left(x^{*}, y^{*}\right) \\
\nabla H\left(x^{*}, y^{*}\right) \\
\nabla g_{\mathcal{I}^{*}}\left(x^{*}, y^{*}\right)
\end{array}\right]
$$

has full column rank $d+s$. Then $\left(x^{*}, y^{*}, u^{*}, v^{*}\right)$ is an M-stationary point of problem (CP) and a local optimal solution of the penalized problem $\left(\mathrm{CP}_{\mu}\right)$ for some $\mu \geq 0$.

Proof. Since LICQ holds at $y^{*}$, the rank of the matrix

$$
S J_{1}^{*}=\left[\begin{array}{ll}
\nabla_{y} h\left(x^{*}, y^{*}\right)^{T} & \nabla_{y} g_{\mathcal{I}^{*} \cup \mathcal{J}^{*}}\left(x^{*}, y^{*}\right)^{T}
\end{array}\right]
$$

equals to $\left|\mathcal{I}^{*}\right|+\left|\mathcal{J}^{*}\right|+n$. Then $r\left(J^{*}\right)=r\left(S J^{*}\right)+r\left(S J_{1}^{*}\right)=d+s+m+n-\left|\mathcal{K}^{*}\right|$ and thus the conclusions in Theorem 5.2 hold.

The following example illustrates the application of Theorem 5.2 and Corollary [5.1.

Example 5.1 Consider the following bilevel program:

$$
\begin{aligned}
\min & F(x, y) \\
\text { s.t. } & x \in[-3,2], \\
& H(x, y):=x^{2}+y-2=0, \\
& y \in S(x):=\underset{y}{\operatorname{argmin}} f(x, y):=y^{3}-3 y \\
\text { s.t. } & g_{1}(x, y):=x-y \leq 0, \\
& g_{2}(x, y):=y-3 \leq 0,
\end{aligned}
$$

where $F(x, y)$ is a Lipschitz continuous function. It is easy to see that the solution set for the lower level program is

$$
S(x)= \begin{cases}\{x\} & \text { if } x \in[-3,-2) \cup(1,2], \\ \{-2,1\} & \text { if } x=-2, \\ \{1\} & \text { if } x \in(-2,1]\end{cases}
$$

and the value function is

$$
V(x)= \begin{cases}x^{3}-3 x & \text { if } x \in[-3,-2) \cup(1,2], \\ -2 & \text { if } x \in[-2,1] .\end{cases}
$$

In fact since the lower level feasible set $Y(x):=\{y \in \mathbb{R}: x \leq y \leq 3\}$ is uniformly bounded whenever $x \in[-3,2]$ and LICQ holds at each $y \in S(x), x \in[-3,2]$, we can conclude that the value function is Lipschitz continuous without actually calculating it. For any $x$, the KKT condition for the lower level problem is

$$
0=3 y^{2}-3-u_{1}+u_{2}, g_{i}(x, y) \leq 0, u_{i} \geq 0, u_{i} g_{i}(x, y)=0, i=1,2 .
$$


Hence the combined problem can be written as follows:

$$
\begin{array}{cl}
\min _{x, y, u} & F(x, y) \\
\text { s.t. } & f(x, y)-V(x) \leq 0, \\
& H(x, y)=0, \\
& \nabla_{y} L(x, y, u):=3 y^{2}-3-u_{1}+u_{2}=0, \\
& x \in[-3,2],(-g(x, y), u) \in \Omega^{2} .
\end{array}
$$

It is easy to see that the feasible region of the bilevel problem contains three points: $\left(x^{*}, y^{*}\right)=$ $(-2,-2),(\hat{x}, \hat{y})=(1,1),(\tilde{x}, \tilde{y})=(-1,1)$. From calculation,

$\nabla\left(\nabla_{y} L\right)(x, y, u)=(0,6 y,-1,1), \nabla H(x, y)=(2 x, 1), \nabla g_{1}(x, y)=(1,-1), \nabla g_{2}(x, y)=(0,1)$.

Suppose that the optimal solution of the bilevel problem is $\left(x^{*}, y^{*}\right)=(-2,-2)$ and the one for the combined program is $\left(x^{*}, y^{*}, u_{1}^{*}, u_{2}^{*}\right)=(-2,-2,9,0)$. The index sets $\mathcal{I}^{*}=\{1\}, \mathcal{K}^{*}=\{2\}$ and $\mathcal{J}^{*}$ is empty. The rank of the matrix

$$
S J^{*}=\left[\begin{array}{c}
\nabla H\left(x^{*}, y^{*}\right) \\
\nabla g_{1}\left(x^{*}, y^{*}\right)
\end{array}\right]=\left[\begin{array}{cc}
-4 & 1 \\
1 & -1
\end{array}\right]
$$

is equal to 2. Hence by Corollary 5.1, $\left(x^{*}, y^{*}, u_{1}^{*}, u_{2}^{*}\right)$ is an M-stationary point of problem (CP).

Suppose that the optimal solution of the bilevel problem is $(\tilde{x}, \tilde{y})=(-1,1)$ and the one for the combined program is $\left(\tilde{x}, \tilde{y}, \tilde{u}_{1}, \tilde{u}_{2}\right)=(-1,1,0,0)$. The index sets $\tilde{\mathcal{K}}=\{1,2\}$ and both $\tilde{\mathcal{I}}, \tilde{\mathcal{J}}$ are empty. The rank of the matrix

$$
J\left(\tilde{x}, \tilde{y}, \tilde{u}_{1}, \tilde{u}_{2}\right)=\left[\begin{array}{c}
\nabla\left(\nabla_{y} f+u \nabla_{y} g\right)(\tilde{x}, \tilde{y}) \\
\nabla H(\tilde{x}, \tilde{y})
\end{array}\right]=\left[\begin{array}{cc}
0 & 6 \\
-2 & 1
\end{array}\right]
$$

is equal to 2. Hence by Theorem 5.2. $\left(\tilde{x}, \tilde{y}, \tilde{u}_{1}, \tilde{u}_{2}\right)$ is an M-stationary point of problem (CP). Moreover $\left(x^{*}, y^{*}, u_{1}^{*}, u_{2}^{*}\right)$ and $\left(\tilde{x}, \tilde{y}, \tilde{u}_{1}, \tilde{u}_{2}\right)$ are local solutions of the penalized problem for some $\mu \geq 0$ :

$$
\begin{array}{rl}
\min _{x, y, u} & F(x, y)+\mu\left((f(x, y)-V(x))_{+}+|H(x, y)|+\left|\nabla_{y} L(x, y, u)\right|+|\min \{-g(x, y), u\}|\right) \\
\text { s.t. } & x \in[-3,2] .
\end{array}
$$

Since RCRCQ is a stronger condition for RCPLD, the following result follows from Theorem 3.1 and Propositions 5.1 and 5.2 .

Theorem 5.3 Let $\left(x^{*}, y^{*}, u^{*}, v^{*}\right)$ be a local solution of $(\mathrm{CP})$. Suppose that either $Y(x)=Y$ is independent of $x$ with $Y$ compact or the set-valued map $Y(x)$ is uniformly bounded around $x^{*}$ and MFCQ holds at $y$ for all $y \in S\left(x^{*}\right)$. Given index sets $\mathcal{I}_{3}, \mathcal{I}_{4} \subseteq \mathcal{J}^{*}$ and $\mathcal{I}_{2} \subseteq I_{G}^{*}, \alpha \in\{0,1\}$, 
denote the matrix

$$
J^{\prime}(x, y, u, v, \alpha, w):=\left[\begin{array}{ccc}
\nabla\left(\nabla_{y} f+u \nabla_{y} g+v \nabla_{y} h\right)(x, y) & \nabla_{y} h(x, y)^{T} & \nabla_{y} g(x, y)^{T} \\
\nabla h(x, y) & 0 & 0 \\
\nabla H(x, y) & 0 & 0 \\
\nabla G_{\mathcal{I}_{2}}(x, y) & 0 & 0 \\
\nabla g_{\mathcal{I}^{*} \cup \mathcal{I}_{3}}(x, y) & 0 & 0 \\
0 & 0 & E_{\mathcal{K}^{*} \cup \mathcal{I}_{4}} \\
\alpha(\nabla f(x, y)-(w, 0)) & 0 & 0
\end{array}\right],
$$

where $E_{\mathcal{K}^{*} \cup \mathcal{I}_{4}} \subseteq \mathbb{R}^{\left(\left|\mathcal{K}^{*}\right|+\left|\mathcal{I}_{4}\right|\right) \times m}$ denotes the matrix with $e_{i}^{T}$ as its rows, $i \in \mathcal{K}^{*} \cup \mathcal{I}_{4}$ and $e_{i} \in \mathbb{R}^{m}$ is the vector such that the $i$-th component is one and others are zero. Assume that the matrix $J^{\prime}\left(x^{*}, y^{*}, u^{*}, v^{*}, \alpha, w^{*}\right)$ where $w^{*} \in c o W\left(x^{*}\right)$ has the same rank with the matrix $J^{\prime}\left(x^{k}, y^{k}, u^{k}, v^{k}, \alpha, w^{k}\right)$ for all sequences $\left\{x^{k}\right\},\left\{y^{k}\right\},\left\{u^{k}\right\},\left\{v^{k}\right\},\left\{w^{k}\right\}$ satisfying $x^{k} \rightarrow x^{*}, y^{k} \rightarrow$ $y^{*}, u^{k} \rightarrow u^{*}, v^{k} \rightarrow v^{*}, w^{k} \rightarrow w^{*}, w^{k} \in \mathrm{coW}\left(x^{k}\right)$. Then RCRCQ for $(C P)$ hold at $\left(x^{*}, y^{*}, u^{*}, v^{*}\right)$ and $\left(x^{*}, y^{*}, u^{*}, v^{*}\right)$ is an $M$-stationary point of problem $(C P)$.

The following example illustrate the result.

Example 5.2 Consider the following bilevel program:

$$
\begin{aligned}
\min & F(x, y) \\
\text { s.t. } & H(x, y):=x_{1}-x_{2}+y-\frac{1}{2}=0, \\
y \in \underset{y}{\operatorname{argmin}} & f(x, y):=x_{1} \exp (y)-x_{2} \exp (y) \\
\text { s.t. } & g_{1}(y):=-y-\ln 2 \leq 0, \\
& g_{2}(y):=y-\ln 2 \leq 0,
\end{aligned}
$$

where $F(x, y)$ is a Lipschitz continuous function. It is easy to see that the solution set for the lower level program is

$$
S(x)= \begin{cases}{[-\ln 2, \ln 2]} & \text { if } x_{1}=x_{2}, \\ \{\ln 2\} & \text { if } x_{1}<x_{2}, \\ \{-\ln 2\} & \text { if } x_{1}>x_{2},\end{cases}
$$

and the value function

$$
V(x)= \begin{cases}0 & \text { if } x_{1}=x_{2}, \\ 2\left(x_{1}-x_{2}\right) & \text { if } x_{1}<x_{2}, \\ \frac{1}{2}\left(x_{1}-x_{2}\right) & \text { if } x_{1}>x_{2} .\end{cases}
$$

In fact we do not need the above explicit representation of the value function. Indeed, since the constraint set $Y=[-\ln 2, \ln 2]$ is compact, by Danskin's theorem, for any $x$ around $x^{*}$ the Clarke subdifferential of the value function is equal to

$$
\partial^{c} V(x)=c o W(x)=c o\left\{\nabla_{x} f\left(x, y_{x}\right) \mid y_{x} \in S(x)\right\}=c o\left\{\exp \left(y_{x}\right) \mid y_{x} \in S(x)\right\}(1,-1) .
$$


The combined problem can be written as follows:

$$
\begin{array}{cl}
\min _{x, y, u} & F(x, y) \\
\text { s.t. } & f(x, y)-V(x) \leq 0, H(x, y)=0, \\
& \nabla_{y} L(x, y, u):=x_{1} \exp (y)-x_{2} \exp (y)-u_{1}+u_{2}=0, \\
& (-g(y), u) \in \Omega^{2} .
\end{array}
$$

It is easy to see that the bilevel program only has three kinds of optimal solutions, $\left(x_{1}^{*}, x_{2}^{*}, y^{*}\right)=$ $\left(a, a, \frac{1}{2}\right)$ with $a \in \mathbb{R},\left(\hat{x}_{1}, \hat{x}_{2}, \ln 2\right)$ with $\hat{x}_{1}<\hat{x}_{2}$ and $\left(\tilde{x}_{1}, \tilde{x}_{2},-\ln 2\right)$ with $\hat{x}_{1}>\hat{x}_{2}$. We now verify that RCRCQ holds in the following three cases.

(a) Consider the optimal solution $\left(x_{1}^{*}, x_{2}^{*}, y^{*}\right)=\left(a, a, \frac{1}{2}\right)$ with $a \in \mathbb{R}$, the corresponding solution for the combined program is $\left(x^{*}, y^{*}, u_{1}^{*}, u_{2}^{*}\right)=\left(a, a, \frac{1}{2}, 0,0\right)$. Obviously, the index sets $\mathcal{K}^{*}=\{1,2\}$ and both $\mathcal{I}^{*}, \mathcal{J}^{*}=\emptyset$. For $\alpha \in\{0,1\}$, denote by

$$
J^{\prime}(x, y, u, \alpha, \omega):=\left[\begin{array}{ccccc}
\exp (y) & -\exp (y) & x_{1} \exp (y)-x_{2} \exp (y) & -1 & 1 \\
1 & -1 & 1 & 0 & 0 \\
0 & 0 & 0 & 1 & 0 \\
0 & 0 & 0 & 0 & 1 \\
\alpha(\exp (y)-w) & -\alpha(\exp (y)-w) & \alpha\left(x_{1} \exp (y)-x_{2} \exp (y)\right) & 0 & 0
\end{array}\right],
$$

where $w \in \operatorname{co}\left\{\exp \left(y_{x}\right) \mid y_{x} \in S(x)\right\}$. It is easy to see that $r\left(J^{\prime}\left(x^{*}, y^{*}, u^{*}, \alpha, w^{*}\right)\right)=r\left(J^{\prime}(x, y, u, \alpha, w)\right)=$ 4 for any $\alpha \in\{0,1\}$, and any $(x, y, u)$ and $w$. Hence $R C R C Q$ holds by Theorem 5.3.

(b) Consider the optimal solution $\left(\hat{x}_{1}, \hat{x}_{2}, \ln 2\right)$ with $\hat{x}_{1}<\hat{x}_{2}$, the corresponding solution for the combined program is $\left(\hat{x}_{1}, \hat{x}_{2}, \ln 2,0, \hat{u}_{2}\right)$ with $\hat{x}_{1}<\hat{x}_{2}$ and $\hat{u}_{2}>0$. The index sets $\hat{\mathcal{K}}=\{1\}$ and $\hat{\mathcal{I}}=\{2\}, \hat{\mathcal{J}}=\emptyset$, and for any $x$ around $\hat{x}=\left(\hat{x}_{1}, \hat{x}_{2}\right), S(x)=\{\ln 2\}, \operatorname{co} W(x)=(2,-2)^{T}$. For $\alpha \in\{0,1\}$, denote by

$$
J^{\prime}(x, y, u, \alpha, w):=\left[\begin{array}{ccccc}
\exp (y) & -\exp (y) & x_{1} \exp (y)-x_{2} \exp (y) & -1 & 1 \\
1 & -1 & 1 & 0 & 0 \\
0 & 0 & 1 & 0 & 0 \\
0 & 0 & 0 & 1 & 0 \\
\alpha(\exp (y)-2) & -\alpha(\exp (y)-2) & \alpha\left(x_{1} \exp (y)-x_{2} \exp (y)\right) & 0 & 0
\end{array}\right] .
$$

It is easy to see that $r\left(J^{\prime}(x, y, u, \alpha, w)\right)=4$ or any $\alpha \in\{0,1\}$ and any $(x, y, u)$ and $w$. Hence RCRCQ holds from Theorem 5.3 .

(c) Consider the optimal solution $\left(\tilde{x}_{1}, \tilde{x}_{2},-\ln 2\right)$ with $\tilde{x}_{1}>\tilde{x}_{2}$, the corresponding solution for the combined program is $\left(\tilde{x}_{1}, \tilde{x}_{2},-\ln 2, \tilde{u}_{1}, 0\right)$ with $\tilde{x}_{1}>\tilde{x}_{2}$ and $\hat{u}_{1}>0$. Similarly as in case (b), RCRCQ holds. 


\section{Acknowlegement}

The authors would like to thank the anonymous referees for their helpful suggestions and comments which help us to improve the presentation of the paper.

\section{References}

[1] R. Andreani, G. Haeser, M.L. Schuverdt and P.J.S. Silva, A relaxed constant positive linear dependence constraint qualification and applications, Math. Program., 135(2012), 255-273.

[2] R. Andreani, J.M. Martínez and M.L. Schuverdt, On the relation between constant positive linear dependence condition and quasinormality constraint qualification, J. Optim. Theory Appl., 125(2005), 473-483.

[3] X. Chen, L. Guo, Z. Lu and J.J. Ye, An augmented Lagrangian method for non-Lipschitz nonconvex programming, SIAM J. Numer. Anal., 55(2017), 168-193.

[4] F.H. Clarke, Optimization and Nonsmooth Analysis, Wiley-Interscience, New York, 1983.

[5] F.H. Clarke, Yu.S. Ledyaev, R.J. Stern and P.R. Wolenski, Nonsmooth Analysis and Control Theory, Springer, New York, 1998.

[6] J.M. Danskin, The Theory of Max-Min and its Applications to Weapons Allocation Problems, Springer, New York, 1967.

[7] S. Dempe, J. Dutta and B.S. Mordukhovich, New necessary optimality conditions in optimistic bilevel programming, Optimization, 56 (2007), 577-604.

[8] S. Dempe and A.B. Zemkoho, The bilevel programming problems: reformulations, constraint qualifications and optimality conditions, Math. Program., 138 (2013), 447-473.

[9] J. Gauvin and F. Dubeau, Differential properties of the marginal function in mathematical programming, Math. Program. Study, 19(1982), 101-119.

[10] L. Guo and G.-H. Lin, Notes on some constraint qualifications for mathematical programs with equilibrium constraints, J. Optim. Theory Appl., 156(2013), 600-616.

[11] L. Guo, G.-H. Lin and J.J. Ye, Second-order optimality conditions for mathematical programs with equilibrium constraints, J. Optim. Theory Appl., 158(2013), 33-64.

[12] L. Guo, G.-H., Lin, J.J. Ye and J. Zhang, Sensitivity analysis of the value function for parametric mathematical programs with equilibrium constraints, SIAM J. Optim., 24(2014), $1206-1237$.

[13] L. Guo and J.J. Ye, Necessary optimality conditions and exact penalization for non-Lipschitz nonlinear programs, Math. Program., 168(2018), 571-598.

[14] L. Guo, J. Zhang and G.-H. Lin, New results on constraint qualifications for nonlinear extremum problems and extensions, J. Optim. Theory Appl., 163(2014), 737-754. 
[15] A.F. Izmailov, M.V. Solodov and E.I. Uskov, Global convergence of augmented Lagrangian methods applied to optimization problems with degenerate constraints, including problems with complementarity constraints, SIAM J. Optim., 22(2012), 1579-1606.

[16] R. Janin, Direction derivative of the marginal function in nonlinear programming, Math. Program. Study, 21(1984), 110-126.

[17] A. Jourani and L. Thibault, The approximate subdifferential of composite functions, Bull. Austral. Math. Soc., 47(1993), 443-455.

[18] C. Kanzow and A. Schwartz, Mathematical programs with equilibrium constraints: enhanced Fritz John-conditions, new constraint qualifications, and improved exact penalty results, SIAM J. Optim., 20(2010), 2730-2753.

[19] L. Minchenko and S. Stakhovski, On relaxed constant rank regularity condition in mathematical programming, Optim., 60(2011), 429-440.

[20] J. Mirrlees, The theory of moral hazard and unobservable behaviour: Part I, Rev. Econ. Stud., 66(1999), 3-22.

[21] B.S. Mordukhovich, Variational Analysis and Generalized Differentiation, I: Basic Theory; II: Applications, Springer, Berlin, 2006.

[22] B.S. Mordukhovich, Variational Analysis and Applications, Springer, New York, 2018.

[23] B.S. Mordukhovich, Bilevel optimization and variational analysis, in Advances in Bilevel Optimization, S. Dempe and A. Zemkoho, eds., arXiv:1907.06140, 2019.

[24] B.S. Mordukhovich, N. M. Nam and H. M. Phan, Variational analysis of marginal functions with applications to bilevel programming, J. Optim. Theory Appl., 152 (2012), pp. 557-586.

[25] J.V. Outrata, On the numerical solution of a class of Stackelberg problems, Z. Oper. Res., 34(1990), 255-277.

[26] L. Qi and Z. Wei, On the constant positive linear dependence condition and its application to SQP methods, SIAM J. Optim., 10(2000), 963-981.

[27] R.T. Rockafellar and R.J.-B. Wets, Variational Analysis, Springer, Berlin, 1998.

[28] X. Wang and Y. Yuan, An augmented Lagrangian trust region method for equality constrained optimization, Optim. Methods soft., 30(2015), 559-582.

[29] J.J. Ye, Constraint qualifications and necessary optimality conditions for optimization problems with variational inequality constraints, SIAM J. Optim., 10(2000), 943-962.

[30] J.J. Ye, Necessary optimality conditions for multiobjective bilevel programs, Math. Opera. Res., 36(2011), 165-184.

[31] J.J. Ye, Constraint qualifications and optimality conditions in bilevel optimization, in Advances in Bilevel Optimization, S. Dempe and A. Zemkoho, eds., arXiv:1910.04072, 2019. 
[32] J.J. Ye and D. Zhu, Optimality conditions for bilevel programming problems, Optim., 33(1995), 9-27.

[33] J.J. Ye and D. Zhu, New necessary optimality conditions for bilevel programs by combining the MPEC and value function approaches, SIAM J. Optim., 20(2010), 1885-1905. 